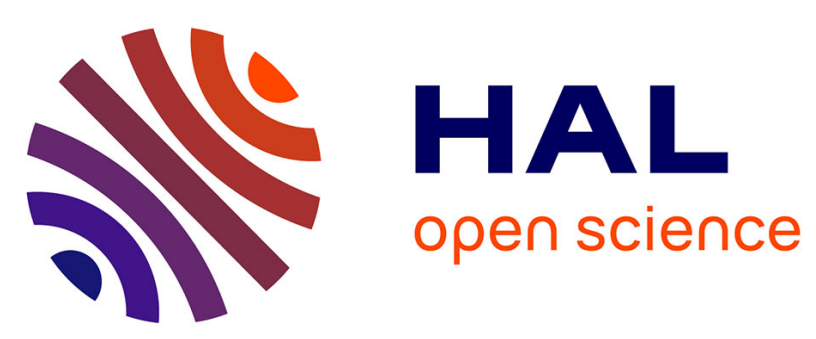

\title{
How well can linear stability analysis predict the behavior of an outward valve brass instrument model?
}

Lionel Velut, Christophe Vergez, Joël Gilbert, Mithra Djahanbani

\section{To cite this version:}

Lionel Velut, Christophe Vergez, Joël Gilbert, Mithra Djahanbani. How well can linear stability analysis predict the behavior of an outward valve brass instrument model?. Acta Acustica united with Acustica, 2017, 103 (1), pp.132-148. 10.3813/AAA.919039 . hal-01245846v4

\section{HAL Id: hal-01245846 \\ https://hal.science/hal-01245846v4}

Submitted on 22 Feb 2017

HAL is a multi-disciplinary open access archive for the deposit and dissemination of scientific research documents, whether they are published or not. The documents may come from teaching and research institutions in France or abroad, or from public or private research centers.
L'archive ouverte pluridisciplinaire HAL, est destinée au dépôt et à la diffusion de documents scientifiques de niveau recherche, publiés ou non, émanant des établissements d'enseignement et de recherche français ou étrangers, des laboratoires publics ou privés. 


\title{
How well can Linear Stability Analysis predict the behaviour of an outward-striking valve brass instrument model?
}

\author{
Lionel Velut ${ }^{1}$, Christophe Vergez ${ }^{1}$, Joël Gilbert ${ }^{2}$, and Mithra Djahanbani ${ }^{1}$ \\ ${ }^{1}$ LMA, CNRS, UPR 7051, Aix-Marseille Univ., Centrale Marseille, F-13453 \\ Marseille cedex 13, France. \\ ${ }^{2}$ Laboratoire d'Acoustique de l'Université du Maine, UMR CNRS-6613, Avenue \\ Olivier Messiaen, 72085 Le Mans cedex 9, France
}

December 13, 2016

\begin{abstract}
A physical model of brass instrument is considered in this paper : a one degree-of-freedom outward-striking valve for the lips, non-linearly coupled to a modal representation of the air column. It is studied through Linear Stability Analysis (LSA) of the equilibrium solution. This approach provides the threshold blowing pressure value, at which instability occurs, and the instability frequency value. The relevance of the results of this method is theoretically limited to the neighbourhood of the equilibrium solution. This paper checks the efficiency of LSA to understand the behaviour of the model computed through time-domain simulations. As expected, a good agreement is observed between LSA and numerical simulations of the complete nonlinear model around the oscillation threshold. For blowing pressures far above the oscillation threshold, the picture is more contrasted. In most of the cases tested, a periodic regime coherent with the LSA results is observed, but over-blowing, quasi-periodicity and period-doubling also occur. Interestingly, LSA predicts the production of the pedal note by a trombone, for which only nonlinear hypotheses have been previously proposed. LSA also predicts the production of a saxhorn note which, although known to musicians, has barely been documented.
\end{abstract}

\section{Introduction}

Linear Stability Analysis (LSA) can be used to analyse the behaviour of dynamical systems around equilibrium points (i.e. non-oscillating solutions). LSA consists in writing a linearised version of a 
dynamical system. The stability of the linearised system is then assessed by studying its response to harmonic perturbations.

LSA has already been applied to physical models of musical instruments, such as woodwind instruments [Wilson and Beavers, 1974, Chang, 1994, Silva et al., 2008, Karkar et al., 2012], flute-like instruments [Terrien et al., 2014] and brass instruments [Cullen et al., 2000, Lopez et al., 2006, Silva et al., 2007]. By definition, the domain of relevance of the LSA results is theoretically limited to the neighbourhood of the equilibrium solution. However, recent results on flutes have highlighted that LSA can predict important features of periodic regimes, such as their frequencies [Terrien et al., 2014]. This paper examines to what extent LSA can be used to understand some aspects of the behaviour of a physical model of brass instrument.

Physical models of brass instuments have been proposed in multiple studies [Eliott and Bowsher, 1982, Fletcher, 1993, Adachi and Sato, 1996, Cullen et al., 2000, Campbell, 2004, Silva et al., 2007]. Since our focus in this study is a simple model, a one degree-of-freedom system is retained to model the player's lips: the outward-striking valve, also referred to as "(+,-)" in some publications. The same goal of simplicity makes us ignore nonlinear propagation in the bore of the instrument, which is responsible for "brassy sounds" at high sound levels [Myers et al., 2012]. The coupling by the airflow blown between the lips and the air column inside the bore is modelled through a usual nonlinear algebraic equation [Hirschberg et al., 1995]. This model is detailed in Section 2.1. Even such a simple brasswind model has more parameters needing to be tuned than the simplest models of woodwind instruments, which is based on two dimensionless parameters only [Hirschberg et al., 1995, Dalmont et al., 1995, Taillard et al., 2010, Bergeot et al., 2013]. However, brasswind players make their instrument oscillate on several modes, which implies a significant modification of the mechanical characteristics of their lips. In musical terms, this corresponds to playing multiple notes without pulling a slide nor depressing a valve, which is part of the playing technique of all brass instruments. Therefore, the lip dynamics cannot be ignored, which implies an increase in the number of parameters to tune. A bibliographical review is given in Section 2.2 to give grounds to the values chosen for each parameter of the model. In Section 2.3, details are given on how LSA is applied to the model. There are several possible approaches to highlighting nonlinear model behaviours to compare them with LSA results. For instance, the Harmonic Balance Method gives a Fourier series approximation of the steady state of periodic regimes, including unstable ones [Gilbert et al., 1989, Cochelin and Vergez, 2009]. Since the pioneering work described in [Schumacher, 1981, McIntyre et al., 1983], it is also possible to carry out time-domain simulations at moderate computational cost, providing access to transients and possibly non-periodic solutions. The second approach is retained here (see Section 2.4). Section 3 compares LSA results and numerical simulations for different sets of parameter values. Periodic regimes, corresponding to the usual sound of the instrument, are explored, along with less common regimes such as quasi-periodicity and period-doubling. In Section 4, we focus on the lowest acoustic resonance of brass instruments, called the pedal note, a particularly interesting 
Figure 1: (Color online) Closed-loop model in free oscillation, suitable for the description of most self-sustained musical instruments. Self-sustained oscillations are generated by the localised nonlinear coupling between a linear exciter and a linear resonator. For brass instruments, the exciter is the lip reed while the resonator is the air column inside the bore, and the coupling is due

case where LSA and numerical simulation results are surprisingly different from other regimes.

\section{Tools}

\section{$2.1 \quad$ Brass instrument model}

In most wind instruments [Fletcher, 1993, Chaigne and Kergomard, 2016], including brass instruments [Eliott and Bowsher, 1982, Yoshikawa, 1995, Cullen et al., 2000], the oscillation results from the coupling between an exciter and a resonator. More generally, the closed-loop system representation shown in Figure 1 has been widely used by the musical acoustics community since the seminal work of Helmholtz [Helmholtz, 1877, McIntyre et al., 1983].

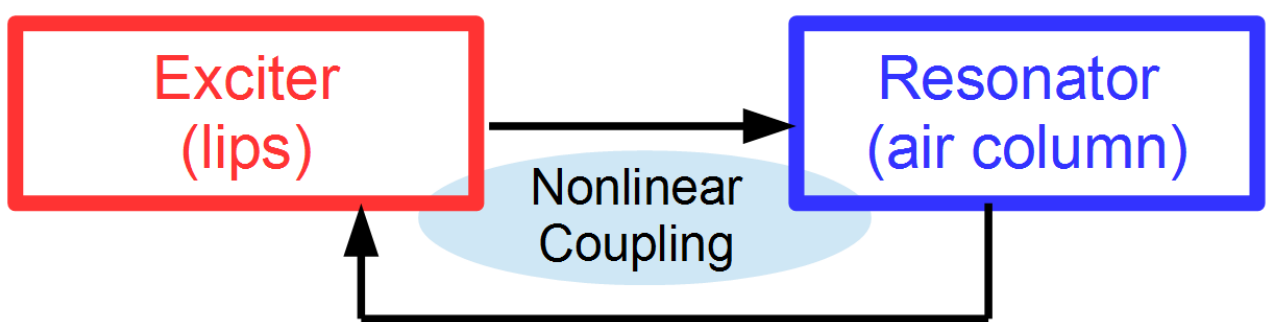
to the air flow between the lips.

For brass instruments, the exciter is the lips of the musician. It is represented by a linear, oscillator-like valve linking the height of the channel between the lips $h(t)$ and the pressure difference across the lips $\delta p(t)=p_{b}-p(t)$, where $p_{b}$ is the blowing pressure, and $p(t)$ is the oscillating pressure signal inside the mouthpiece (the input of the bore).

A one degree of freedom valve (referred to hereafter as "1-DOF valve") [Fletcher, 1993] is enough to model the lips for common playing situations [Yoshikawa, 1995] with a manageable number of parameters. Two kinds of 1-DOF valves can be considered : the "outward-striking" valve tends to open when $\delta p$ grows, while the "inward-striking" valve tends to close.

While it is now admitted that woodwind reeds can be satisfactorily modelled by inward striking valves [Wilson and Beavers, 1974, Dalmont et al., 1995], there is no consensus about the modelling of the lip reed, as neither the outward-striking nor the inward-striking valve model reproduces all the behaviours observed with real musicians. Particularly, brass players are able to reach a playing frequency $f_{\text {osc }}$ above and below the $n^{\text {th }}$ bore resonance frequency $f_{a c, n}$ [Campbell, 2004], while a 1-DOF inward-striking or outward-striking valve model is limited to playing frequencies respectively below or above $f_{a c, n}$ to meet the regeneration condition explained in [Eliott and Bowsher, 1982]. Moreover, measurements of the mechanical response of artificial [Cullen et al., 2000, Neal et al., 2001] and natural lips [Newton et al., 2008] revealed the 
coexistence of both inward-striking and outward-striking resonances - this coexistence allowing $f_{\text {osc }}$ to be below or above $f_{a c, n}$.

However, situations where $f_{\text {osc }}$ is below $f_{a c, n}$ (inward-striking behaviour) are mostly specific to some musical effects. For normal playing situations, the playing frequency is above $f_{a c, n}$, and an outward-striking valve model is preferred. Moreover, the geometry of human lips makes them open when the pressure in the mouth increases, which is consistent with the behaviour of the outwardstriking valve model. The relevance of this choice will be reinforced throughout this article, by comparing the results of the model analysis with experimental behaviours of brasswinds.

The outward-striking valve model gives the relation below, linking the height of the channel between the lips and the pressure difference across the lips :

$$
\frac{d^{2} h}{d t^{2}}+\frac{\omega_{l}}{Q_{l}} \frac{d h}{d t}+\omega_{l}^{2}\left(h-h_{0}\right)=\frac{1}{\mu}\left(p_{b}-p(t)\right),
$$

where $\omega_{l}=2 \pi f_{l}\left(\mathrm{rad} \cdot \mathrm{s}^{-1}\right)$ is the lip resonance angular frequency; $Q_{l}$ the (dimensionless) quality factor of the lips; $h_{0}$ the value of $h(t)$ at rest; $\mu$ a lip surface mass equivalent $\left(\mathrm{kg} \cdot \mathrm{m}^{-2}\right)$. The variables are reported on the sketch of the lip region in Figure 2:

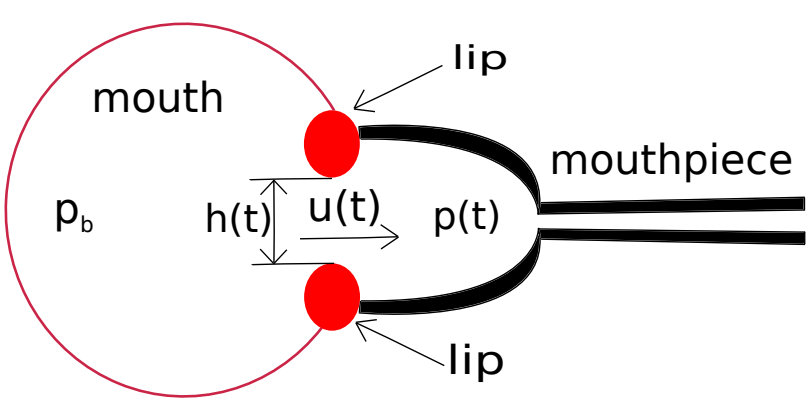

Figure 2: (Colour online) Sketch of the mouth and lips of the musician and the instrument mouthpiece. The mouth (left) is considered as a cavity under a static pressure $p_{b}$. The lips (ellipses) separate the mouth from the mouthpiece. The height between the lips is $h(t)$, the airflow between the lips is $u(t)$ and the pressure in the mouthpiece is $p(t)$.

This model assumes the mouth pressure to be constant, even though the existence of an oscillating component in the mouth has been demonstrated experimentally [Fréour and Scavone, 2013]. A more precise model would consider this oscillating component, which is due to the tunable pipe formed by the vocal tract [Eliott and Bowsher, 1982]. A significant role of the vocal tract has been shown for saxophone and clarinet playing [Clinch et al., 1982, Fritz, 2005, Scavone et al., 2008, Guillemain et al., 2010, Chen et al., 2011]. However, for brass instrument playing, the role of the vocal tract does not seem to be significant when playing periodic regimes in the usual musical range of the instrument - although its interaction with the lips has been highlighted by experimental studies [Kaburagi et al., 2011, Chen et al., 2012, Fréour and Scavone, 2013, Fréour et al., 2015, Boutin et al., 2015].

The resonator is the air column inside the bore of a trombone or a saxhorn (see Section 4.2). It is modelled by its input impedance, which is the ratio between pressure $P(\omega)$ and acoustic flow 
$U(\omega)$ in the mouthpiece. Its expression in the frequency domain is:

$$
Z(\omega)=\frac{P(\omega)}{U(\omega)} .
$$

Nonlinear effects in the resonator should be taken into account to accurately describe the behaviour of brass instruments at medium/high playing levels [Hirschberg et al., 1996, Myers et al., 2012] particularly the "brassy sound" related to the formation of shock waves. However, the main objective of this work is the study of oscillation at low levels. Therefore the acoustic propagation along the bore is assumed to be linear and thus the input impedance fully describes the resonator in our model. Here, input impedances of a Courtois "T149" tenor trombone (and when mentioned, a Couesnon "Excelsior" baritone-saxhorn in $B b$ ) are used. Impedances are measured with the impedance sensor described in [Macaluso and Dalmont, 2011]. They are fitted by a sum of complex modes (pole-residue functions) using a Least Mean Squares method, as described in [Silva, 2009, p.28-40]. The characteristic impedance of the resonator is $Z_{c}=\rho c / S, S$ being the input cross section of the bore at the mouthpiece rim. The modal-fitted impedance is written:

$$
Z(\omega)=Z_{c} \sum_{n=1}^{N}\left[\frac{C_{n}}{j \omega-s_{n}}+\frac{C_{n}^{*}}{j \omega-s_{n}^{*}}\right],
$$

$s_{n}$ and $C_{n}$ being the complex poles and the complex residues of the $n^{\text {th }}$ complex mode, respectively. Translation of eq. (3) in the time domain and decomposition of $p(t)$ into its modal components $p_{n}$, such as $p(t)=2 . \sum_{n=1}^{N} \operatorname{Re}\left(p_{n}\right)$ results in an ordinary differential equation for each $p_{n}$ :

$$
\frac{d p_{n}}{d t}=Z_{c} \cdot C_{n} \cdot u(t)+s_{n} \cdot p_{n} \quad \forall n \in[1, N]
$$

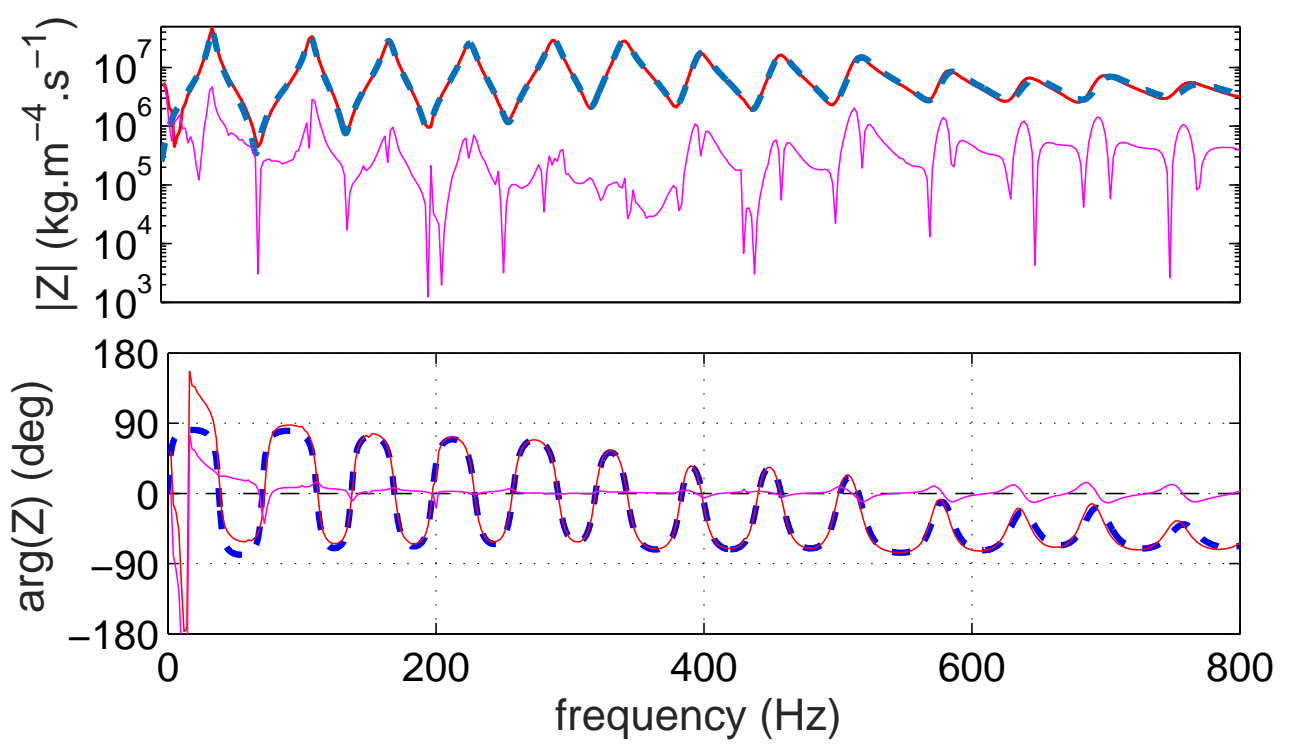

Figure 3: (colour online) Magnitude (top) and phase (bottom) of the input impedance of a Courtois tenor trombone with the slide in its first position. The dashed (blue) curve depicts the measured impedance, the solid (red) curve is the fitted curve with 18 complex modes. The difference between fit and measurement is also plotted (magenta). 
The measured trombone impedance and an 18-mode fit are compared, cf. Figure 3. The maximum relative difference between the measured and the fitted curves, for frequencies above $30 \mathrm{~Hz}$, is lower than $2.6 \%$ for the magnitude, and $4.7 \%$ for the phase. Measurement in low frequency is limited by the impedance sensor precision.

Those two linear elements (exciter and resonator) are non-linearly coupled by the airflow through the lip channel. The air jet is assumed to be laminar in the lip channel, but turbulent in the mouthpiece, all its kinetic energy being dissipated without any pressure recovery. Applying the Bernoulli law and the mass conservation law gives the following expression of the flow between lips, depending on the pressure difference and the height of the lip channel [Wilson and Beavers, 1974, Eliott and Bowsher, 1982, Hirschberg et al., 1995]:

$$
u(t)=\sqrt{\frac{2}{\rho}} W h(t) \sqrt{p_{b}-p(t)},
$$

where $u(t)$ is the airflow $\left(\mathrm{m}^{3} \cdot \mathrm{s}^{-1}\right), h(t)$ the height of the channel between the lips $(\mathrm{m}), \rho=1.19$ $\mathrm{kg} \cdot \mathrm{m}^{-3}$ the density of the air at $20{ }^{\circ} \mathrm{C}$ and $W$ the width of the lip channel $(\mathrm{m})$.

The dynamics of the system described by (5), (1) and (4) can be put into a state-space representation $\dot{X}=F(X)$, where $\mathrm{F}$ is a nonlinear vector function, and $\mathrm{X}$ the state vector, containing the observables of the system. Since $p(t)=\sum_{n=1}^{N} 2 \operatorname{Re}\left(p_{n}(t)\right)$, this results in the following system:

$$
\left\{\begin{array}{l}
\frac{d^{2} h(t)}{d t^{2}}=-\omega_{l}^{2} h(t)-\frac{\omega_{l}}{Q_{l}} \frac{d h(t)}{d t}-\frac{p(t)}{\mu}+\omega_{l}^{2} h_{0}+\frac{p_{b}}{\mu} \\
\frac{d p_{n}}{d t}=s_{n} p_{n}(t)+Z_{c} C_{n} \sqrt{\frac{2}{\rho}} W h(t) \sqrt{p_{b}-p(t)} \text { for } n \in[1, N] .
\end{array}\right.
$$

This leads to the following state vector, similar to the one proposed in [Silva et al., 2014]:

$$
X=\left[h(t) ; \frac{d h}{d t} ;\left\{p_{n}(t), n \in[1, N]\right\}\right]^{\prime},
$$

and the function $F$ can be written as:

$$
\frac{d X}{d t}=\left(\begin{array}{c}
\frac{d h}{d t} \\
\frac{d^{2} h}{d t^{2}} \\
\frac{d p_{1}}{d t} \\
\vdots \\
\frac{d p_{n}}{d t}
\end{array}\right)=F(X)=\left(\begin{array}{c}
X(2) \\
-\omega_{l}^{2} X(1)-\frac{\omega_{l}}{Q_{l}} X(2)-\frac{1}{\mu} \sum_{k=3}^{N+2} 2 \operatorname{Re}[X(k)]+\omega_{l}^{2} h_{0}+\frac{p_{b}}{\mu} \\
s_{1} X(3)+C_{1} \cdot Z_{c} \cdot \sqrt{\frac{2}{\rho}} W X(1) \sqrt{p_{b}-\sum_{k=3}^{N+2} 2 \operatorname{Re}[X(k)]} \\
\vdots \\
s_{N} X(N+2)+C_{N} \cdot Z_{c} \cdot \sqrt{\frac{2}{\rho}} W X(1) \sqrt{p_{b}-\sum_{k=3}^{N+2} 2 \operatorname{Re}[X(k)]}
\end{array}\right) .
$$

\subsection{Choice of lip parameters}

Setting the values for the parameters of the lip model is not obvious, because measuring the mechanical admittance (velocity over force ratio) under playing conditions (oscillating lips) seems out of reach, even if some experiments tend to it [Newton et al., 2008]. Adjusting parameters to 
161

\begin{tabular}{|c|c|c|c|c|c|c|}
\hline Reference & $h_{0}(\mathrm{~m})$ & $W(\mathrm{~m})$ & $f_{l}(\mathrm{~Hz})$ & $\mu^{-1}\left(\mathrm{~m}^{2} \cdot \mathrm{kg}^{-1}\right)$ & $Q_{l}$ & Summary \\
\hline [Eliott and Bowsher, 1982] & $\mathrm{N} / \mathrm{A}$ & $\mathrm{N} / \mathrm{A}$ & 200 & 0.2 & 0.5 & $Q_{l}$ measured on cheek \\
\hline [Cullen et al., 2000] & & & & & & $1^{s t}$ (Outward) mode \\
\hline Embouchure: Soft & $6.3 \cdot 10^{-4}$ & $18 \cdot 10^{-3}$ & 189 & 0.07 & 10.5 & Artificial lips \\
\hline Medium & $5.3 \cdot 10^{-4}$ & $12 \cdot 10^{-3}$ & 203.5 & 0.11 & 6 & 3 embouchures \\
\hline Tight & $4.4 \cdot \cdot 10^{-4}$ & $11 \cdot 10^{-3}$ & 222 & 0.09 & 9 & \\
\hline [Lopez et al., 2006] & $2 \cdot 10^{-4}$ & $30 \cdot 10^{-3}$ & 162 & 0.03 & 5 & Artificial lips \\
\hline [Gazengel et al., 2007] & & & & & & Human lips; \\
\hline Embouchure: Soft & $\mathrm{N} / \mathrm{A}$ & $\mathrm{N} / \mathrm{A}$ & 115.7 & $\mathrm{~N} / \mathrm{A}$ & 0.79 & saxophone-like \\
\hline $167 \quad$ Medium & $\mathrm{N} / \mathrm{A}$ & $\mathrm{N} / \mathrm{A}$ & 479.9 & $\mathrm{~N} / \mathrm{A}$ & 0.46 & position; \\
\hline Tight & $\mathrm{N} / \mathrm{A}$ & $\mathrm{N} / \mathrm{A}$ & 1073 & $\mathrm{~N} / \mathrm{A}$ & 0.46 & 3 embouchures \\
\hline [Newton et al., 2008] & $\mathrm{N} / \mathrm{A}$ & $\mathrm{N} / \mathrm{A}$ & 32 & $\mathrm{~N} / \mathrm{A}$ & $1.2-1.8$ & $\begin{array}{c}\text { Human lips } \\
\text { High-speed camera }\end{array}$ \\
\hline [Richards, 2003] & $5 \cdot 10^{-4}$ & $7 \cdot 10^{-3}$ & 162 & 0.19 & 3.7 & $\begin{array}{c}\text { Artificial lips } \\
\text { fit for good results }\end{array}$ \\
\hline [Rodet and Vergez, 1996] & $\mathrm{N} / \mathrm{A}$ & $\mathrm{N} / \mathrm{A}$ & 428.4 & 0.67 & 2.88 & $\begin{array}{l}\text { Trumpet; adjusted } \\
\text { for simulation }\end{array}$ \\
\hline [Adachi and Sato, 1996] & $1 \cdot 10^{-3}$ & $7 \cdot 10^{-3}$ & $60-700$ & variable & $0.5-3$ & $\begin{array}{c}\text { Trumpet; adjusted } \\
\text { for simulation }\end{array}$ \\
\hline
\end{tabular}

get results comparable with measured signals does not seem a good approach: Indeed, even though a one-DOF model depends on a small number of parameters, different sets of parameter values may lead to similar results [Hélie et al., 1999]. Moreover, lip valve parameters are expected to vary far more than reed valve parameters, particularly the lip resonance frequencies.

A bibliographical review on lip parameter values has been done. Results from the literature are gathered in Table 1 along with a brief summary of the method used in the reviewed articles.

Table 1: Review of different values of lip parameters from literature, along with a brief explanation of the method. In some articles, certain values are not available (N/A). For papers presenting 2DOF lip models, only the first, outward-striking DOF is reported. All but the last two references

deal with trombone parameter values.

This work complements the review published in [Newton, 2009, p.119]. Many authors do not provide the parameter values they use, nor do they give explanations about their method to get these values, except the fact that these parameters allow periodic self-sustained oscillation of the model. Measurements on human or artificial lips were made in conditions as similar as possible to the playing conditions. The list of publications is not exhaustive: we left aside most of the publications since they do not justify their values or do not fit their measurements with a modal lip-reed model.

Geometric parameters (lip channel width, and lip channel height when the player is not blowing) given in all studies are consistent, around $W=12 \cdot 10^{-3} \mathrm{~m}$ and $h_{0}=5 \cdot 10^{-4} \mathrm{~m}$. Parametric studies 
have shown that variations in these values do not drastically change the qualitative behaviour of the model : numerical values change but the overall behaviour is the same. Similar observations have been made about $\mu$, even though the range of the values gathered is a little wider $(\mu \in[5,33]$ for the trombone).

Measurements from [Gazengel et al., 2007, Newton et al., 2008] tend to give low quality-factor values between 0.5 and 2 for human lips. However, preliminary analysis carried out with $Q_{l} \approx 1$ showed very unrealistic pressure thresholds (order of magnitude : $10^{4}$ to $10^{5} \mathrm{~Pa}$ ). Thus, a value for $Q_{l}=7$ was chosen, closer to the values measured on artificial lips $\left(Q_{l} \in[3.7,10.5]\right)$. The set of parameters used for simulation and LSA throughout this paper is given in Table 2:

\begin{tabular}{|c|c|c|c|}
\hline$h_{0}(\mathrm{~m})$ & $W(\mathrm{~m})$ & $1 / \mu\left(\mathrm{m}^{2} \mathrm{~kg}^{-1}\right)$ & $Q_{l}$ \\
\hline $5.10^{-4}$ & $12.10^{-3}$ & 0.11 & 7 \\
\hline
\end{tabular}

Table 2: Lip parameters retained in this study.

The value of $f_{l}$ is constantly adapted by the musician while playing. For this reason, we performed LSA with $f_{l}$ values ranging from $20 \mathrm{~Hz}$ to $500 \mathrm{~Hz}$. This allows oscillation on the first eight regimes of the instrument, which correspond to the usual notes of the trombone, from $B b 1$ to $B b 4$ with the slide in first position.

\subsection{Stability of the equilibrium solution}

Linearising a closed-loop system to assess potential instabilities is a widely used method, in the dynamical systems community [Bergé et al., 1995] as well as in musical acoustics for brasswind, woodwind and flute-like instruments [Wilson and Beavers, 1974, Cullen et al., 2000, Silva et al., 2008, Auvray et al., 2012, Terrien et al., 2014]. Basically, the equations modelling the system are linearised around a known equilibrium solution. Then, the stability of this solution is determined.

When the system described in Section 2.1 is in static equilibrium, the lip opening position has a static value $h(t)=h_{e}$. This equilibrium position is slightly larger than the lip opening at rest $h_{0}$, due to the constraint of the blowing pressure on the inner sides of the lips. Similarly, there is a small static overpressure $p_{e}$ at the input of the bore of the instrument, as $Z(\omega=0)$ is nonzero. This is related to the pressure loss in the instrument. Mathematically, this equilibrium is obtained by cancelling all time derivatives in the system, as described in appendix A. The value of $A=\sqrt{p_{b}-p_{e}}$ is obtained by solving:

$$
A^{3}+\frac{A^{2}}{\beta}+h_{0} \mu \omega_{l}^{2} A-\frac{p_{b}}{\beta}=0
$$

with $\beta=\frac{W Z(\omega=0)}{\mu \omega_{l}^{2}} \sqrt{\frac{2}{\rho}}$. The value of $Z(\omega=0)$ is extrapolated from the fitted version of the impedance. Equation (9) has 1 or 3 real roots. In the latter case, the smallest real positive root should be considered to compute $p_{e}=p_{b}-A^{2}$ [Silva, 2009], as $Z(\omega=0)$ is small. The lip channel height at equilibrium $h_{e}$ is then given by (1) with $\ddot{h}=\dot{h}=0$. 
In the vicinity of the equilibrium solution $X_{e}$, the linearised function $\tilde{F}$ can be written as:

$$
\tilde{F}(X)=F\left(X_{e}\right)+J_{F}\left(X_{e}\right)\left(X-X_{e}\right)
$$

where $J_{F}(X)$ is the Jacobian matrix of the function $F$ and $X_{e}$ the state vector at the equilibrium solution. The solutions of $\dot{X}=\tilde{F}(X)$ are under the form :

$$
X(t)-X_{e}=\sum_{i=1}^{N} U_{i} e^{\lambda_{i} \cdot t}
$$

where $\lambda_{i}$ are the eigenvalues of $J_{F}\left(X_{e}\right)$ and $U_{i}$ the corresponding eigenvectors.

Thus, the eigenvalues of the Jacobian matrix give information about the stability of the equilibrium solution for a given set of parameters. If at least one of these eigenvalues $\lambda$ has a positive real part, the amplitude of the linearised solution tends to infinity while time increases, which means the equilibrium is unstable and the solution starts oscillating. Referring to (11), this means that one of the terms of the sum dominates the solution, all other terms being decreasing exponentials. As a first approximation, the solution of the linearised system can be written:

$$
X(t)-X_{e}=\sum_{\operatorname{Re}\left(\lambda_{i}\right)>0} U_{i} e^{\lambda_{i} \cdot t}
$$

The developed tool finds the lowest value of $p_{b}$ at which the equilibrium solution becomes unstable, i.e. the value at which one eigenvalue $\lambda$ with positive real part appears. This value of $p_{b}$ is further referred to as $p_{\text {thresh }}$ the oscillation threshold (or threshold pressure). During the transient phase of the oscillation, the exponential growth of the amplitude is determined by the positive real part of $\lambda$, and the angular frequency is given by its imaginary part $\omega=\operatorname{Im}(\lambda)$. However, the nonlinearities of the system limit the final amplitude and also affect the oscillation frequency of the steady state. This method only detects instabilities emerging from the equilibrium solution. If a stable oscillating regime coexists along with the stable equilibrium solution, it will not be detected. This situation occurs for example in certain woodwind instruments, where the Hopf bifurcation (connecting the equilibrium solution to the oscillating one) is inverse in some cases [Grand et al., 1997, Dalmont et al., 2000, Farner et al., 2006, Ricaud et al., 2009].

\subsection{Time-domain simulation}

Another approach for studying musical instruments relies on time-domain $a b$ initio simulations of the chosen model, for a given set of parameters.

Multiple numerical methods have been developed to simulate wind instruments with models similar to the one presented in Section 2.1. Various approaches have been proposed to implement the resonator acoustic behaviour. The reflection function of the bore has been widely used [Schumacher, 1981, McIntyre et al., 1983, Adachi and Sato, 1995, Vergez and Rodet, 1997, Gilbert and Aumond, 2008]. The modal decomposition of the bore has been chosen for this arti- 
cle, and computations are carried out with the open-source MoReeSC software tool, freely available [MoReeSC, 2013]. Its principles and results have been described in [Silva et al., 2014]. This simulation tool uses the state-space paradigm, similar to the one presented in Section 2.1. It allowed us to simulate the behaviour of the model with a high number of acoustic modes for the resonator (18 in this study), and offers the necessary flexibility to modify the model parameters, including the resonator parameters, as it is done in Section 4.

\section{Results}

\subsection{Linear Stability Analysis}

The LSA method detailed in Section 2.3 is applied to the model defined in Section 2.1, with the set of lip parameters defined in Table 2. The resonator is modelled with a modal fit $(\mathrm{N}=18$ in Equation (3)) of a measured impedance ( $B b$ trombone, first position).

For each value of $f_{l}$ considered, the eigenvalues of the Jacobian matrix $J_{F}\left(X_{e}\right)$ presented in Equation (10) are computed for increasing values of $p_{b}$, until a first instability, characterized by at least one eigenvalue with positive real part, occurs. For each value of $f_{l}$, Figure 4 a represents $p_{\text {thresh }}$, the lowest value of $p_{b}$ giving rise to an unstable equilibrium solution (see section 2.3). Figure $4 \mathrm{~b}$ represents the imaginary part of the corresponding eigenvalue divided by $2 \pi$, which is the oscillation frequency at threshold, further called $f_{\text {thresh }}$. Each horizontal dotted line in Figure $4 \mathrm{~b}$ represents the $n^{\text {th }}$ acoustic resonance frequency of the instrument $f_{a c, n}$, given by the local maximum of the input impedance amplitude.

It should be noted that, for $p_{b}$ values higher than $p_{\text {thresh }}$, other pairs of conjugate eigenvalues may have a positive real part, which implies a system with multiple instabilities. If different oscillating solutions are stable with these parameters, the system is able to start oscillating on different acoustic resonances. In Figure 4, and later fig. 11 and fig. 14, the first instability (the one corresponding to $p_{b}=p_{\text {thresh }}$ ) is shown for each $f_{l}$ value (solid curve). The second instability is reported only for a narrow range of $f_{l}$ (dashed curve).

Between 20 and $500 \mathrm{~Hz}$, the two curves of Figure 4 can be divided into 8 parts. Each part corresponds to a range of $f_{l}$ associated to one regime of oscillation, related to one acoustic resonance of the instrument: [30,63 Hz] (first regime), [72, $123 \mathrm{~Hz}]$ (second regime), [124, $179 \mathrm{~Hz}],[180,234$ $\mathrm{Hz}],[235,288 \mathrm{~Hz}],[289,352 \mathrm{~Hz}],[353,404 \mathrm{~Hz}]$, [405, $460 \mathrm{~Hz}$. In Figure 4b, an oscillating frequency plateau is maintained just above each value of $f_{a c, n}$. This is the usual behaviour of an outwardstriking valve coupled to an air column: when playing on the $n^{\text {th }}$ acoustic mode of the bore, the oscillation frequency at threshold $f_{\text {thresh }}$ is just above $f_{a c, n}$, which is the resonance frequency of the $n^{\text {th }}$ acoustic mode [Campbell, 2004]. For each regime, $f_{\text {thresh }}$ monotonously follows the variation of $f_{l}$. This matches the experience of the brass player, who can slightly "bend" the pitch up and down, i.e. increase or decrease the pitch, by adjusting $f_{l}$ through the muscular tension of the lips, and by adapting the blowing pressure to the change in $p_{t h r e s h}$. The range of each plateau, i.e. the 
attainable frequency range on each acoustic resonance, has analytical limits depending on the lip quality factor $Q_{l}$, as detailed in [Silva et al., 2007]. These frequency limits are plotted as plain (blue) lines on Fig. 4b). Between $64 \mathrm{~Hz}$ and $71 \mathrm{~Hz}$, the equilibrium solution is unconditionally stable whatever the value of $p_{b}$ : this frequency range covers the impedance minimum between $1^{\text {st }}$ and $2^{\text {nd }}$ peaks, which are farther apart from one another than the other peaks due to the first peak inharmonicity.

It can be observed in Figure 4a that the oscillation threshold globally increases with the rank of the acoustic resonance. A larger $p_{b}$ value is required to reach the higher notes of the instrument, in accordance with the musical experience. For each regime, the $p_{\text {thresh }}$ curve is U-shaped, as already observed in [Silva et al., 2007]. Its minimum value $p_{o p t, n}$, marked with a circle in Figure 4 , is known to depend significantly on the quality factor of the lips $Q_{l}$. In the following, we assume as in [Lopez et al., 2006] that $p_{o p t, n}$ and the associated lip resonance frequency $f_{l, n}^{o p t}$ and oscillation frequency at threshold $f_{\text {thresh }, n}^{\text {opt }}$ represent the optimal playing configuration for a human performer. This hypothesis is in line with what musicians report, i.e. they develop a strategy to minimize the effort to produce a sound on a given regime. The values of $p_{\text {opt }, n}$, between $500 \mathrm{~Pa}$ and $15.3 \mathrm{kPa}$ have the same order of magnitude as blowing pressure measured by [Bouhuys, 1968] and [Fréour, 2013]. The pressure threshold increases faster when $f_{l}$ grows above $f_{l, n}^{\text {opt }}$ rather than when it decreases below $f_{l, n}^{o p t}$, as illustrated by the inset in Figure 4a. These results are compatible with the experience of brass players, who report that "bending down" a note requires less effort than bending it up. The rest of this section focuses on some examples of $\left[p_{b}, f_{l}\right]$ points to illustrate the different behaviours observed with the model. For each case, the agreement between LSA results and the sound produced by the time-domain simulation described in Section 2.4 is discussed. 

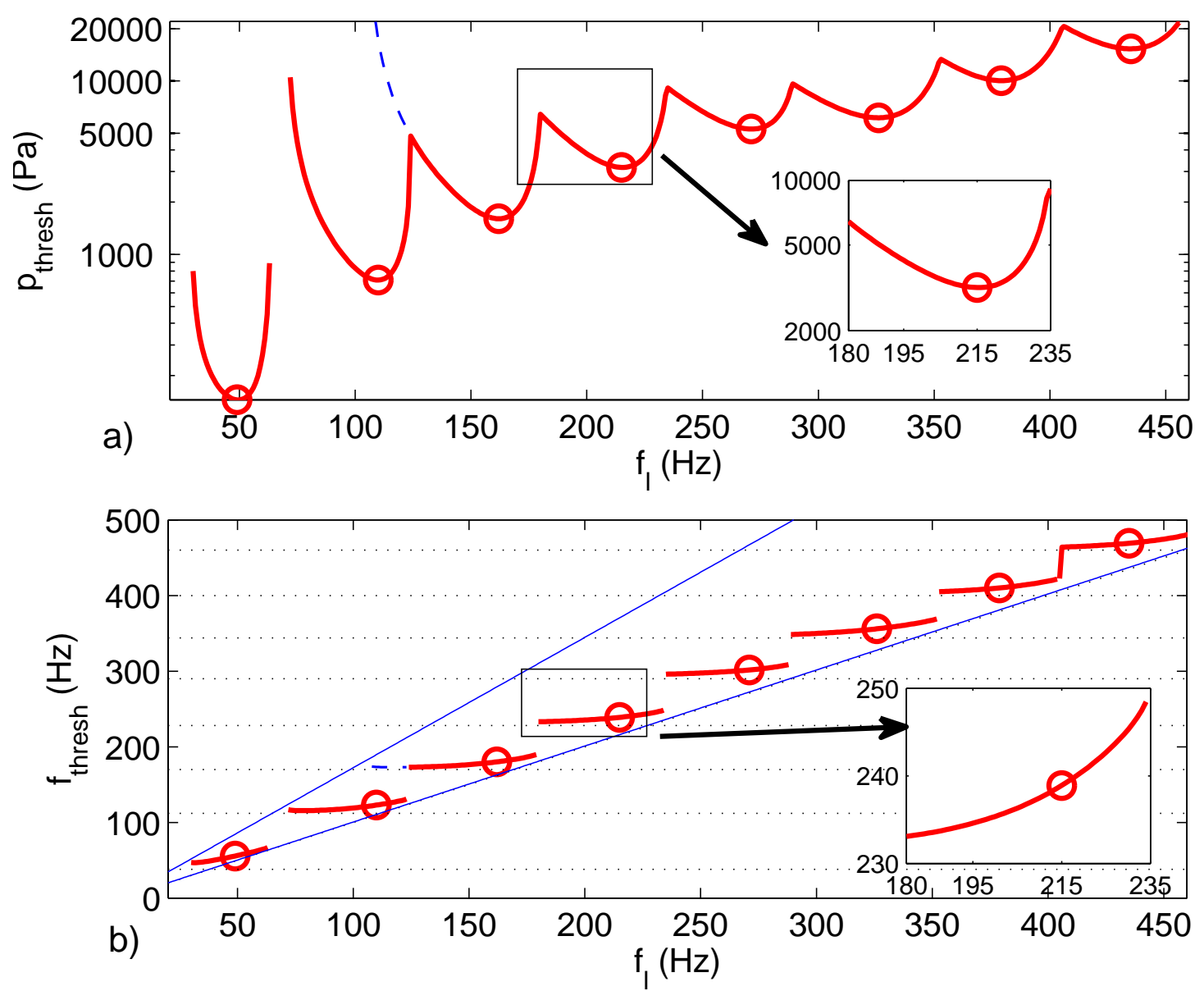

Figure 4: (colour online) Results of LSA applied to the model detailed in Section 2.1 with parameters from Table 2. For a range of lip resonance frequencies $f_{l}$, (a) shows the threshold pressure $p_{\text {thresh }}$, while (b) shows the corresponding oscillation frequency $f_{\text {thresh }}$. Dotted lines are the values of $f_{a c, n}$. Circles indicate the "optimal" values $p_{\text {opt }, n}$ and $f_{\text {thresh }, n}^{\text {opt }}$ as defined in the text. The magnified subplot (zoom on $4^{\text {th }}$ regime) highlights the asymmetrical $p_{\text {thresh }}$ behaviour above and below $p_{o p t, n}$. For illustration, the second destabilisation threshold (a) and the corresponding frequency (b) are also plotted between $f_{l}=109 \mathrm{~Hz}$ and $123 \mathrm{~Hz}$. Diagonal solid (blue) lines in (b) are analytical limits to $f_{\text {thresh }}$ for a lossless model.

\subsection{Exact match between simulation and LSA}

The simulated pressure at the input of the instrument is compared with the LSA results. In particular, the oscillation threshold is assessed by performing simulations with $p_{b}$ in the vicinity of $p_{\text {thresh }}$. The corresponding frequencies, called $f_{\text {osc }}$, are also compared to $f_{\text {thresh }}$ given by LSA. This latter quantity is measured by applying a zero-crossing algorithm [Wall, 2003], with a sliding Hanning window (width $0.3 \mathrm{~s}$, overlapping 99\%). This method results in small computation artefacts, which should not be taken into account.

A simulation with the exact value of $p_{\text {thresh }}$ would theoretically lead to an infinite transient time, defined as the time it takes to reach steady state. Therefore, values of $p_{b}$ slightly below and above $p_{\text {thresh }}$ are tested. To illustrate a periodic oscillation of the model, the lip resonance frequency is 
a)

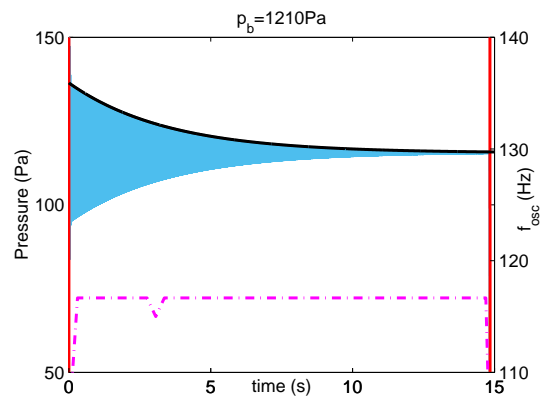

b)

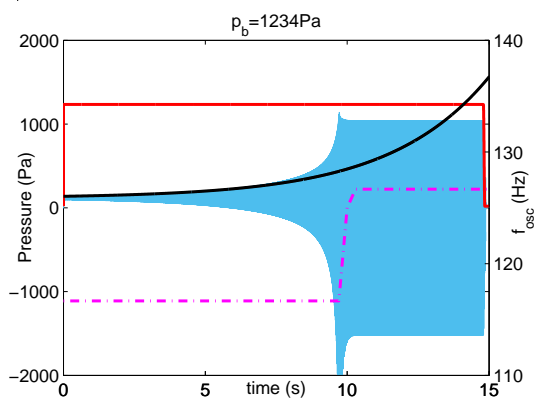

c)

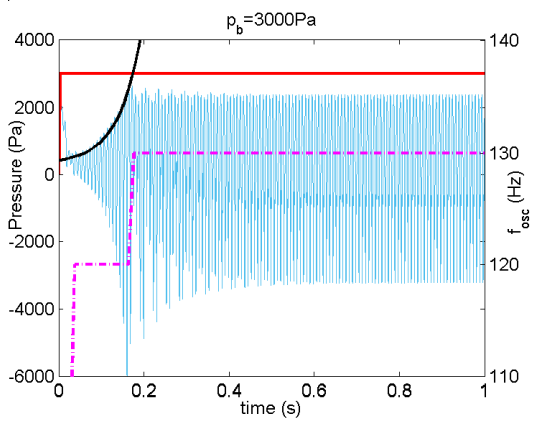

Figure 5: (colour online) Time-domain simulations with parameters from Table 2 and $f_{l}=90 \mathrm{~Hz}$, with mouth pressure $p_{b}$ (horizontal solid line) lower (a) and higher (b) than the linearised model threshold $\left(p_{\text {thresh }}=1222 \mathrm{~Pa}\right)$. Mouth pressure (steady) and mouthpiece pressure (oscillating) are plotted (left vertical axis) along with the exponential growth/diminution of amplitude calculated using LSA (thick curves: envelope of Equation (12)). The dash-dotted curve depicts the instantaneous playing frequency (right vertical axis). The expected oscillation frequency at threshold is $f_{\text {thresh }}=116 \mathrm{~Hz}$. The third plot (c) corresponds to a blowing pressure much higher than the threshold ( $p_{b}=3 \mathrm{kPa}$; zoom on first second of signal).

As expected, the behaviour of time-domain simulations is accurately predicted by LSA as long as $p_{b}$ remains in the vicinity of $p_{\text {thresh }}$ (Figure $5 \mathrm{a}$ and $5 \mathrm{~b}$ ). The value of $p_{\text {thresh }}$ given by LSA is in agreement with simulations. The eigenvalue with the largest real part predicts the frequency and the amplitude of the oscillation at the beginning of the simulation. However, above the pressure threshold in Fig. 5b, after $t=8 \mathrm{~s}$, the simulated amplitude gets affected by nonlinear phenomena 
and is no longer exponential. Thus, this linearised tool provides relevant information about the signal, but is obviously unable to fully predict the amplitude of the sustained regime waveform. The third plot shows the results with $p_{b}=3 \mathrm{kPa}$ much higher than $p_{\text {thresh. }}$. LSA and time-domain simulation give roughly coherent information. As in Figure 5b, the oscillating frequency of the established regime $f_{\text {osc }}=130.5 \mathrm{~Hz}$ is $8 \%$ higher than $\operatorname{Im}(\lambda) /(2 \pi)=120.8 \mathrm{~Hz}$. The difference is 134 musical cents, larger than a semitone. This difference is lower when $p_{b}$ is closer to $p_{t h r e s h}$. Despite this difference, $f_{\text {thresh }}$ predicts which acoustical resonance supports the oscillation. An in vivo experiment has also shown that the pitch rises when the player increases the blowing pressure [Campbell and Greated, 1994]. However, this remark should be considered carefully because during practice a brass player always apply correlated control over mouth pressure and lip muscular activity.

\begin{tabular}{|c|c|c||c|c|}
\hline$p_{b}(\mathrm{~Pa})$ & $\operatorname{Re}(\lambda)$ & $\operatorname{Im}(\lambda) / 2 \pi$ & $f_{\text {osc }}(\mathrm{Hz})$ & measured transient duration $(\mathrm{s})$ \\
\hline 1234 & 0.2864 & 116.74 & 126.5 & 9.71 \\
1500 & 5.5591 & 117.66 & 127.6 & 0.74 \\
2000 & 12.0262 & 118.99 & 128.9 & 0.31 \\
2500 & 16.0891 & 120.01 & 129.7 & 0.215 \\
3000 & 18.8507 & 120.82 & 130.5 & 0.1675 \\
\hline
\end{tabular}

Table 3: Values of the real part of the destabilising eigenvalue $\lambda$, its imaginary part divided by $2 \pi$, the oscillation frequency of the established regime, and the duration of the transient (both measured on simulations) for different values of the blowing pressure (all other parameters unchanged). The real part of $\lambda$ increases with $p_{b}$, which implies a faster-growing envelope as $p_{b}$ increases. This is consistent with the transient duration measured with MIRonsets ${ }^{1}$ function estimating the time needed to reach $95 \%$ of the maximum amplitude of $p(t)$.

Transient time, i.e. the time needed for the amplitude to reach $95 \%$ of its final value, have been measured with different values of $p_{b}$. The values are reported in Table 3.

The transient time decreases while $\operatorname{Re}(\lambda)$ increases, which can be modelled: according to Eq. (12) the amplitude grows exponentially with $\operatorname{Re}(\lambda)$. Thus, under the assumption that $p_{e}$ is negligible compared to $95 \%$ of the final amplitude (hereinafter noted $p_{95 \%}$ ), one can write:

$$
p_{95 \%}=B \cdot e^{\operatorname{Re}(\lambda) \cdot \operatorname{transient}}
$$

where $B$ is a real constant and transient the transient time (s).

Furthermore, according to [Bergé et al., 1995, p.40] in the vicinity of a direct Hopf bifurcation, the maximum amplitude of the oscillation is proportional to the square root of the difference between the parameter value and the threshold value, which means $\sqrt{p_{b}-p_{\text {thresh }}}$ here. Therefore, the value of the pressure at $t=$ transient is:

\footnotetext{
${ }^{1}$ Part of MIRtoolbox: https://www.jyu.fi/hum/laitokset/musiikki/en/research/coe/materials/ mirtoolbox/. Accessed 2016-09-13
} 


$$
p_{95 \%}=0.95 . C \cdot \sqrt{p_{b}-p_{\text {thresh }}},
$$

where $\mathrm{C}$ is a real constant.

Introducing this expression of $p_{95 \%}$ in the natural logarithm of Eq. (13) results in the following analytical expression of the transient time where $A=\frac{0.95 . C}{B}$ :

$$
\text { transient }=\frac{1}{\operatorname{Re}(\lambda)} \cdot \ln \left(A \sqrt{p_{b}-p_{\text {thresh }}}\right) .
$$

With $A=4.75$ fitted on values measured on time-domain simulations, this model matches very well with the evolution of transient durations measured on simulations with different values of $p_{b}$, as shown in Figure 6.

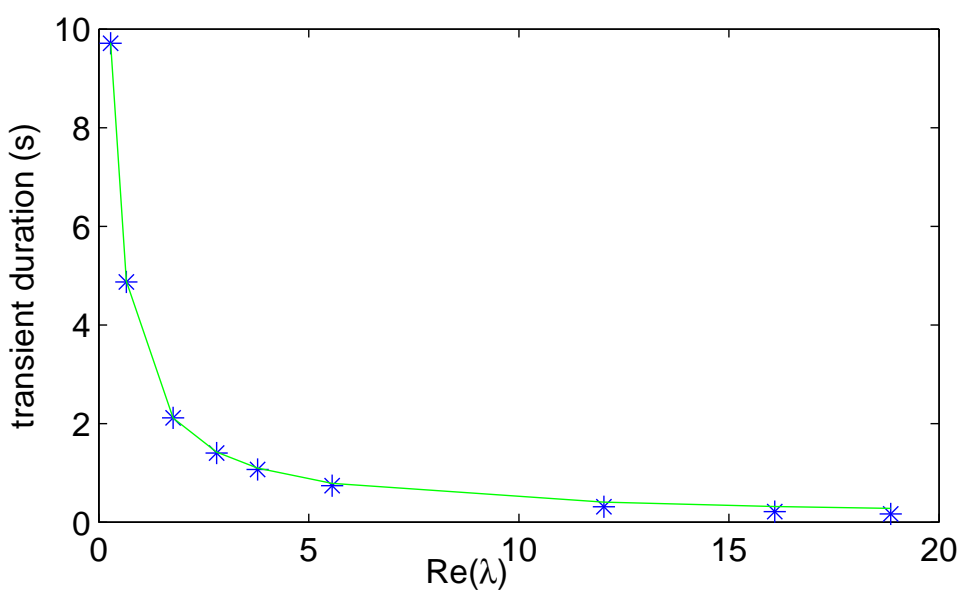

Figure 6: (colour online) Transient durations measured on time-domain simulations, plotted along the $\operatorname{Re}(\lambda)$ value (* symbols). The solid line is the transient duration model described by Eq.(15).

The oscillation frequency also increases with $p_{b}$. An estimate of the frequency is also given (imaginary part of $\lambda$ divided by $2 \pi$ ) which matches well the pseudo-frequency of the transient phase of each signal.

This example is representative of most cases tested: LSA correctly predicts whether the solution is oscillating, with an acceptable estimation of the oscillation frequency. The transient duration can be accurately predicted with the real part of $\lambda$, as described in Eq. (15) even for $p_{b}$ far above the threshold. However, the accuracy of the oscillation frequency prediction is limited, and LSA can predict neither the steady-state waveform nor the nature of the oscillation regime. This latter observation will be further highlighted in the following sub-section.

\subsection{Unforeseen behaviours}

LSA provides a lot of relevant information about the oscillation threshold and the transient phase. This is particularly true when $p_{b}$ is near $p_{\text {thresh. }}$. However, some simulations (detailed below) 
a)

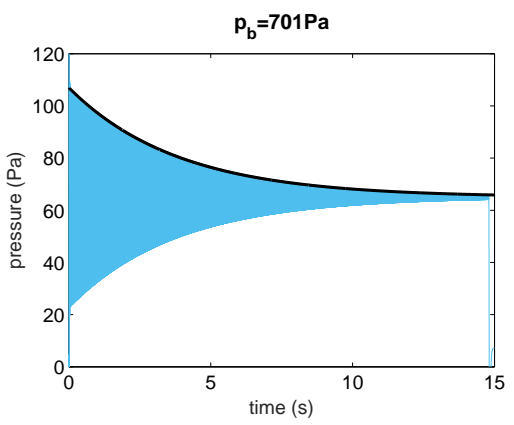

d)

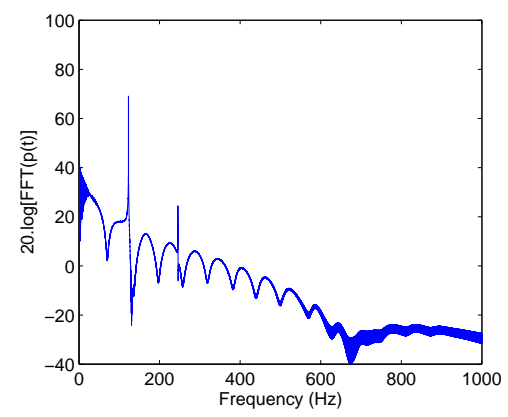

b)

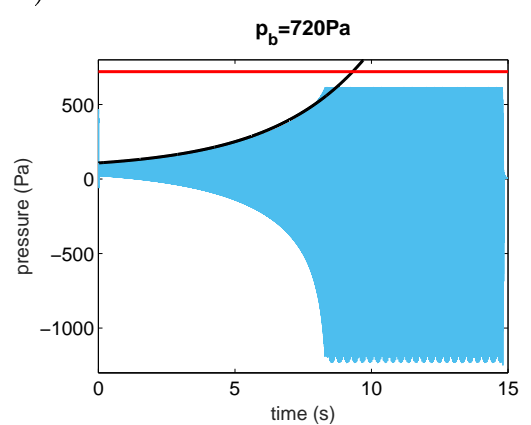

e)

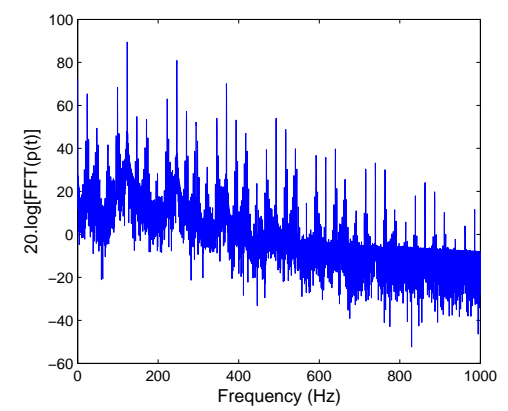

c)

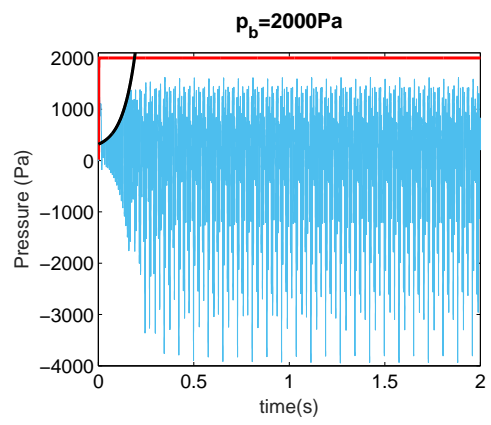

f)

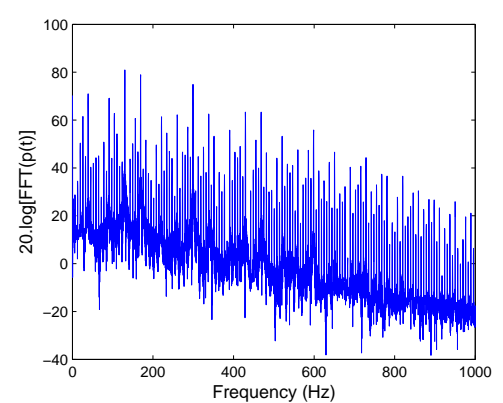

Figure 7: (colour online) Simulation results for $f_{l}=110 \mathrm{~Hz}$, the pressure threshold being $p_{\text {thresh }}=$ $711 \mathrm{~Pa}$. Like in Figure 5 three simulations are shown with $p_{b}=701 \mathrm{~Pa}(\mathrm{a}), p_{b}=720 \mathrm{~Pa}$ (b) and $p_{b}=2 \mathrm{kPa}$, much higher than $p_{\text {thresh }}(\mathrm{c}) \cdot p_{b}$ is plotted as an horizontal solid (red) line. The envelope of Eq. (11) is plotted in plain (black) line. Other parameters (lip characteristics) are given in Table 2. Figures (d), (e) and (f) are the spectra corresponding to (a), (b) and (c), respectively ((e) and (f) calculated using steady regimes of (b) and (c). 
This illustrates the aforementioned limitation of LSA. The existence of an oscillating solution is attested in the vicinity of the bifurcation, and the pressure threshold $p_{\text {thresh }}$ is accurately predicted, but the occurrence of a quasi-periodic regime cannot be predicted.

\section{Period doubling}

When $f_{l}$ is equal to $55 \mathrm{~Hz}, p_{b}$ to $400 \mathrm{~Pa}$ ( $p_{\text {thresh }}$ being $161 \mathrm{~Pa}$ ), and the other parameters are the values given in Table 2 , the simulation result oscillates at $f_{\text {osc }}=32.5 \mathrm{~Hz}$, far below $f_{\text {thresh }}=59.78$ Hz. This is a peculiar behaviour, as this oscillation frequency is significantly under the trombone first acoustic resonance $\left(f_{a c, 1}=38 \mathrm{~Hz}\right)$. Indeed, the chosen model induces playing frequencies above the acoustic resonance frequency $\left(f_{o s c}>f_{a c, n}\right)$, at least near the pressure threshold, to comply with the regeneration condition [Eliott and Bowsher, 1982].

Figure 8 compares the spectrum of the mouthpiece pressure simulated with the aforementioned parameters and $f_{l}=55 \mathrm{~Hz}$ (dotted line) and then with $f_{l}=50 \mathrm{~Hz}$ (solid line). For $f_{l}=50 \mathrm{~Hz}$, $f_{\text {osc }}=65 \mathrm{~Hz}$ is higher than $f_{\text {thresh }}=56.3 \mathrm{~Hz}$, like in previous simulations in Section 3.2. For $f_{l}=55 \mathrm{~Hz}$, a reasonable expectation would be an oscillation frequency slightly higher than $65 \mathrm{~Hz}$, as $f_{\text {osc }}$ tends to increase with $f_{l}$. However, the simulation oscillation frequency at $f_{l}=55 \mathrm{~Hz}$ is $f_{\text {osc }}=32.47 \mathrm{~Hz}$, close to half of its value at $f_{l}=50 \mathrm{~Hz}$.

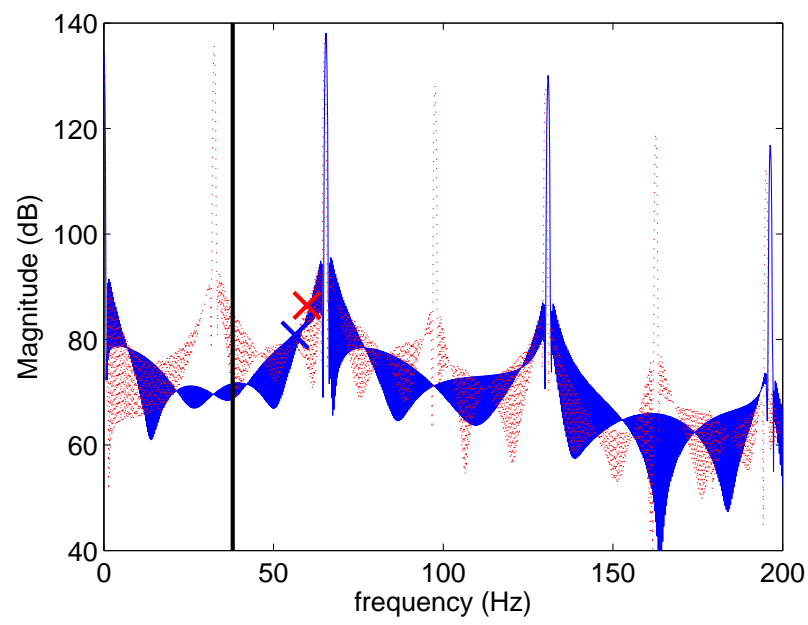

Figure 8: (colour online) Spectra of the simulated trombone mouthpiece pressures, with $p_{b}=400$ $\mathrm{Pa}$ for both lip resonance frequencies, $f_{l}=50 \mathrm{~Hz}$ (solid) and $f_{l}=55 \mathrm{~Hz}$ (dotted) (other parameters from Table 2). Cross markers give the values of $f_{\text {thresh }}=56.3 \mathrm{~Hz}$ for $f_{l}=50 \mathrm{~Hz}$ and $f_{\text {thresh }}=59.8$ $\mathrm{Hz}$ for $f_{l}=55 \mathrm{~Hz}$. The solid vertical line indicates the first acoustic resonance frequency of the trombone bore, $f_{a c, 1}=38 \mathrm{~Hz}$.

Further simulations were carried out, with $f_{l}$ going from 50 to $61 \mathrm{~Hz}$ in steps of $1 \mathrm{~Hz}, p_{b}=400 \mathrm{~Pa}$ and the others parameters set as in Table 2. Table 4 reports the oscillation frequency measured on the simulated signals, along with the $f_{\text {thresh }}$ value predicted by LSA. Between 54 and $55 \mathrm{~Hz}$, the oscillation frequency is almost halved. Then, between 56 and $57 \mathrm{~Hz}$, the frequency is again halved, becoming a quarter of its value for $f_{l}<55 \mathrm{~Hz}$. For $f_{l}=59 \mathrm{~Hz}$ and above, the fundamental 
frequency rises sharply to a value close to its original value, but the energy is far more distributed 421 in the spectrum.

\begin{tabular}{|c||c|c|c|c|c|c|c|c|c|c|c|c|}
\hline$f_{l}(\mathrm{~Hz})$ & 50 & 51 & 52 & 53 & 54 & 55 & 56 & 57 & 58 & 59 & 60 & 61 \\
$422 f_{\text {osc }}(\mathrm{Hz})$ & 65.45 & 65.48 & 65.49 & 65.49 & 65.46 & 32.53 & 32.54 & 16.32 & 16.32 & 65.1 & 65.1 & 65.1 \\
\hline$f_{\text {thresh }}(\mathrm{Hz})$ & 56.3 & 56.97 & 57.71 & 58.36 & 59.08 & 59.78 & 60.51 & 61.27 & 62 & 62.77 & 63.58 & 64.44 \\
\hline
\end{tabular}

Table 4: Oscillation frequencies measured on the simulated mouthpiece pressure, for lip frequencies from 50 to $61 \mathrm{~Hz}, p_{b}=400 \mathrm{~Pa}$ and other parameters from Table 2. Oscillation frequencies at threshold given by LSA are also reported.

These results are close to those reported in [Gibiat and Castellengo, 2000], with a trombone player performing two successive period doublings. When increasing $f_{l}$ in this range, the model undergoes multiple period-doubling bifurcations. Similar scenarios have been observed on numerical models of woodwind instruments [Gibiat, 1988, Kergomard et al., 2004]. This succession of period doublings is also known as subharmonic cascade or Feigenbaum scenario and leads to chaotic behaviour, which may explain the noisiness of signals above $f_{l}>58 \mathrm{~Hz}$. Again, explaining the occurrence of such phenomena is out of reach with LSA.

\section{Overblowing}

Besides these two nonlinear phenomena, other differences between eigenvalue-based LSA and timedomain simulation can be observed. Another example is given with $f_{l}=120 \mathrm{~Hz}$, the parameters given in Table 2 and a high blowing pressure: $p_{b}=6.5 \mathrm{kPa}$ while the threshold is $p_{\text {thresh }}=1056 \mathrm{~Pa}$. While $f_{\text {thresh }}=128.4 \mathrm{~Hz}$ is just above the $2^{\text {nd }}$ acoustic resonance frequency of the bore $\left(f_{a c, 2}=112\right.$ $\mathrm{Hz}$ ), the simulation oscillation frequency exceeds the $3^{r d}: f_{o s c}=187.5 \mathrm{~Hz}>f_{a c, 3}=170 \mathrm{~Hz}$. Figure 9 shows the spectrum of a simulation oscillating on the third acoustic resonance, while the predicted oscillation at threshold corresponds to the second one.

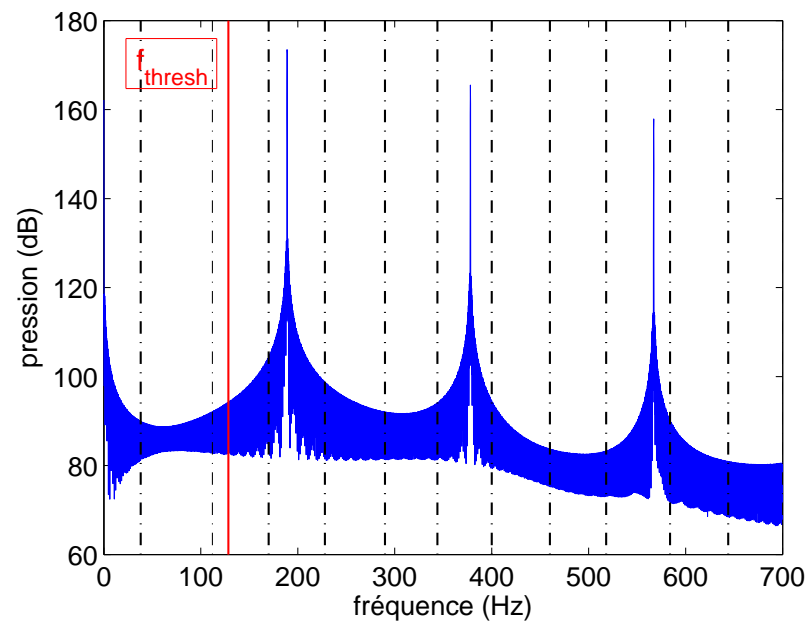

Figure 9: (colour online) Spectrum of simulated mouthpiece pressure for $f_{l}=120 \mathrm{~Hz}$ and $p_{b}=6.5$ $\mathrm{kPa}$ with other parameters taken from Table 2 . The self-sustained oscillation occurs at $f_{\text {osc }}=187.5$ $\mathrm{Hz}$, corresponding to the third acoustic resonance, while LSA predicts an oscillation at $f_{\text {thresh }}=$ $128.4 \mathrm{~Hz}$ (thick vertical line) with $p_{\text {thresh }}=1056 \mathrm{~Pa}$. Each dash-dotted line represents the $n^{\text {th }}$ acoustic resonance frequency $f_{a c, n}$ of the trombone bore. 
The method previously used, which consists in retaining the lowest $p_{b}$ value causing a destabilisation, does not predict the behaviour of the system with such a high blowing pressure. Yet, this oscillation on the third regime can be understood, since another pair of eigenvalues of the Jacobian matrix with a positive real part appears for $p_{b}>p_{\text {thresh }}$. The dashed lines in Figure 4a and $4 \mathrm{~b}$ shows the pressure threshold corresponding to the second pair of such eigenvalues (called $\lambda_{2}$ and $\lambda_{2}^{*}$ ), and the associated oscillation frequency. For $f_{l}=120 \mathrm{~Hz}$ the second threshold is $6116 \mathrm{~Pa}$ with an oscillation frequency equal to $\operatorname{Im}\left(\lambda_{2}\right) / 2 \pi=172 \mathrm{~Hz}$, corresponding to the third regime of oscillation of the system. This is consistent with the behaviour observed in the numerical simulation.

\subsection{Open-loop transfer function}

For a better understanding of the origin of the different instabilities, another LSA formalism is used, which gives visual information about the stability margins of the different oscillation regimes. It consists in studying a linearised version of the open-loop transfer function (OLTF) of the system defined by Equation (5), (1) and (3) [Saneyoshi et al., 1987, Ferrand et al., 2010]. This OLTF is divided into two parts: the exciter admittance $Y_{a}$ which describes the lip reed behaviour, from Equation (5) and (1), and the resonator input impedance, which is modelled with a modal fit of its input impedance $Z$ like in the other formalism (see Equation (3)).

The linearisation of the exciter admittance $Y_{a}$ simplifies to a $1^{\text {st }}$ degree Taylor expansion of Equation (5) near the equilibrium point; Equation (1) is then put into the result. Details can be found in Appendix B about the calculation which leads to the following expression of $Y_{a}$ :

$$
Y_{a}=W h_{e} \sqrt{\frac{2 \delta p_{e}}{\rho}}\left(\frac{D(\omega)}{K h_{e}}-\frac{1}{2 \delta p_{e}}\right),
$$

where $D(\omega)$ represents the dynamics of the lip reed.

The stability of the OLTF, called $H_{O L}$, is then evaluated with the Barkhausen criterion [Wangenheim, 2011], which points to possibly unstable solutions when $H_{O L}=Y_{a} \cdot Z=1$. On a Bode diagram, points with $H_{O L}$ having a $0 \mathrm{~dB}$ magnitude and $0^{\circ}$ phase are limits of stability. This method has already been used for clarinet models with inward-striking valves, and for brass and flute-like instruments [Saneyoshi et al., 1987, Ferrand et al., 2010, Terrien et al., 2014].

Figure 10 shows the Bode diagram of the OLTF of the system fed with the same parameters as in Figure 9. The stability limits are indicated with crosses. 


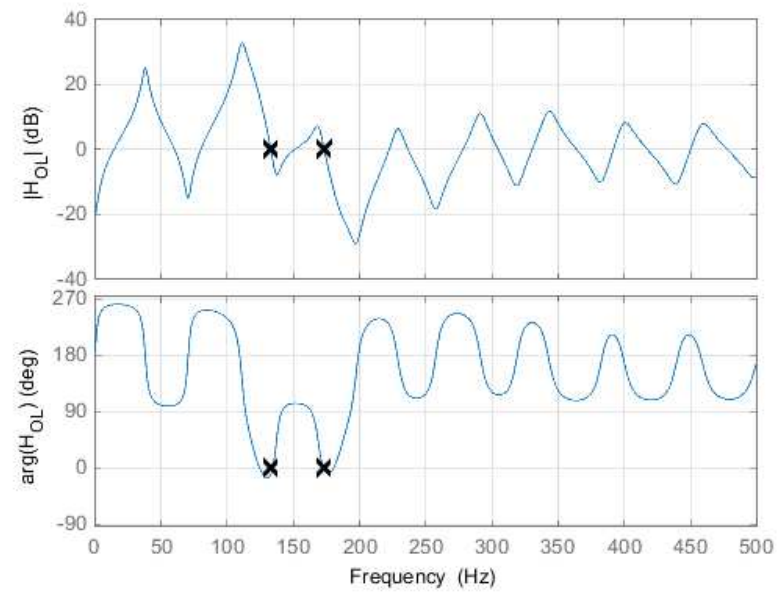

Figure 10: (colour online) Bode diagram of the open-loop transfer function of the trombone model with the parameters specified in Table $2, f_{l}=120 \mathrm{~Hz}$ and $p_{b}=6.5 \mathrm{kPa}$. There are two instability points (crosses), with a $0 \mathrm{~dB}$ magnitude and a zero phase.

Here, the Bode diagram shows two points of $0 \mathrm{~dB}$ magnitude and 0 degree phase at $132 \mathrm{~Hz}$ and $172 \mathrm{~Hz}$. In terms of the eigenvalues-based LSA tool described in Section 2.3, these frequencies correspond to the imaginary part of the eigenvalues of $J_{F}$ calculated with $p_{b}=6500 \mathrm{~Pa}$ and having a positive real part. The frequency obtained with OLTF differs from the one obtained with eigenvalues of the Jacobian matrix, because $f_{\text {thresh }}=128 \mathrm{~Hz}$ is obtained at $p_{b}=p_{\text {thresh }}=1056$ $\mathrm{Pa}$ while the OLTF value is obtained with $p_{b}=6.5 \mathrm{kPa}$. The real part of the second destabilising pair of eigenvalues becomes positive above $6116 \mathrm{~Pa}$, which is compatible with an oscillation on this regime at $p_{b}=6.5 \mathrm{kPa}$. The related frequency at threshold is $172.9 \mathrm{~Hz}$ corresponding to an oscillation on the third acoustic resonance.

Both LSA methods show multiple instabilities of the static solution, that is, multiple possible regimes of oscillation. The predictions of threshold pressures and possible oscillation frequencies are satisfactory. But they give no information either about the stability of these oscillation regimes, or about which regime the instrument will actually oscillate on. This is determined by initial conditions and by the stability of the different oscillating solutions, which depends on nonlinear elements out of reach of the method.

\section{Lowest regime of oscillation}

This chapter focuses on the results of LSA and time-domain simulation on the lowest regime, related to the first acoustic resonance of the air column inside the bore. This lowest playable note is called "pedal note" by musicians. For the trombone in first position, and the saxhorn with no valve depressed (neutral position), the pedal note is a $B b 1$ at $58 \mathrm{~Hz}$ in the musical scale. 


\subsection{The Trombone "pedal note"}

To compare the behaviour of the different registers of the trombone, the ratio between the threshold frequency $f_{\text {thresh }}$ and the resonance frequency of the corresponding acoustical mode $f_{a c, n}$ is computed. Figure $11 \mathrm{a}$ and $11 \mathrm{~b}$ give $p_{\text {thresh }}$ and $f_{\text {thresh }}$ like in Figure 4 but on a smaller $f_{l}$ range, and Figure $11 \mathrm{c}$ gives the $f_{\text {thresh }} / f_{a c, n}$ ratio.

At the lip frequencies corresponding to the pressure threshold minima, called $f_{l, n}^{o p t}$ (see circles in Figure 11), this ratio appears to be significantly higher for the first acoustic resonance than for the other ones: $f_{\text {thresh }, 1}^{\text {opt }} / f_{a c, 1}=55.6 / 38=1.46$ while $f_{\text {thresh }, n}^{\text {opt }} / f_{a c, n} \in[1.04,1.1]$ for $n \geq 2$ as shown in Table 5.

It can be noted that, at least for the five lowest resonances, $f_{\text {thresh }, n}^{\text {opt }}$ is in good agreement with the note supposed to be played on the instrument for this resonance, according to the tempered scale (see Table 5). Therefore, LSA gives a reliable estimation of the note corresponding to these acoustic resonances, including the pedal note, with a relative error between $f_{\text {thresh }, n}^{o p t}$ and the tempered scale note smaller than $5.5 \%$. However, $f_{\text {thresh }, n}^{\text {opt }}$ underestimates the playing frequency of the pedal note while it overestimates the other notes.

\begin{tabular}{|c|c|c|c|c||c|c|}
\hline Regime & $f_{l, n}^{\text {opt }}(\mathrm{Hz})$ & $f_{\text {thresh }, n}^{\text {opt }}(\mathrm{Hz})$ & tempered scale $(\mathrm{Hz})$ & relative error & $f_{a c, n}(\mathrm{~Hz})$ & $f_{\text {thresh }, n}^{\text {opt }} / f_{a c, n}$ \\
\hline 1 & 49 & 55.6 & 58.27 & $-4.6 \%$ & 38 & 1.46 \\
2 & 110 & 122.9 & 116.54 & $5.4 \%$ & 112 & 1.1 \\
3 & 162 & 180.0 & 174.81 & $2.9 \%$ & 170 & 1.06 \\
4 & 215 & 238.9 & 233.08 & $2.5 \%$ & 228 & 1.05 \\
5 & 271 & 301.6 & 291.35 & $3.5 \%$ & 290 & 1.04 \\
\hline
\end{tabular}

Table 5: $f_{\text {thresh }, n}^{\text {opt }}$ values for the five lowest regimes of the trombone, compared with the frequency of the expected note. The acoustic resonance frequency of the corresponding mode, the $f_{l, n}^{o p t}$ value and the $f_{\text {thresh }, n}^{o p t} / f_{a c, n}$ ratio are also given. $f_{\text {thresh }, n}^{\text {opt }}$ is a suitable prediction of the played note. The $f_{\text {thresh }, n}^{o p t} / f_{a c, n}$ ratio is particularly high for the first oscillation regime.

For illustration, a simulation is carried out with the usual parameters from Table 2 with $f_{l}=$ $f_{l, n}^{o p t}=49 \mathrm{~Hz}$ and $p_{b}=150 \mathrm{~Pa}$ ( $p_{\text {thresh }}$ being $\left.146 \mathrm{~Pa}\right)$. The resulting signal oscillates at $f_{\text {osc }}=61.86$ $\mathrm{Hz}$, far higher than $f_{a c, 1}$ : the frequency results of LSA and of simulation are consistent for these parameters as well. 
a)
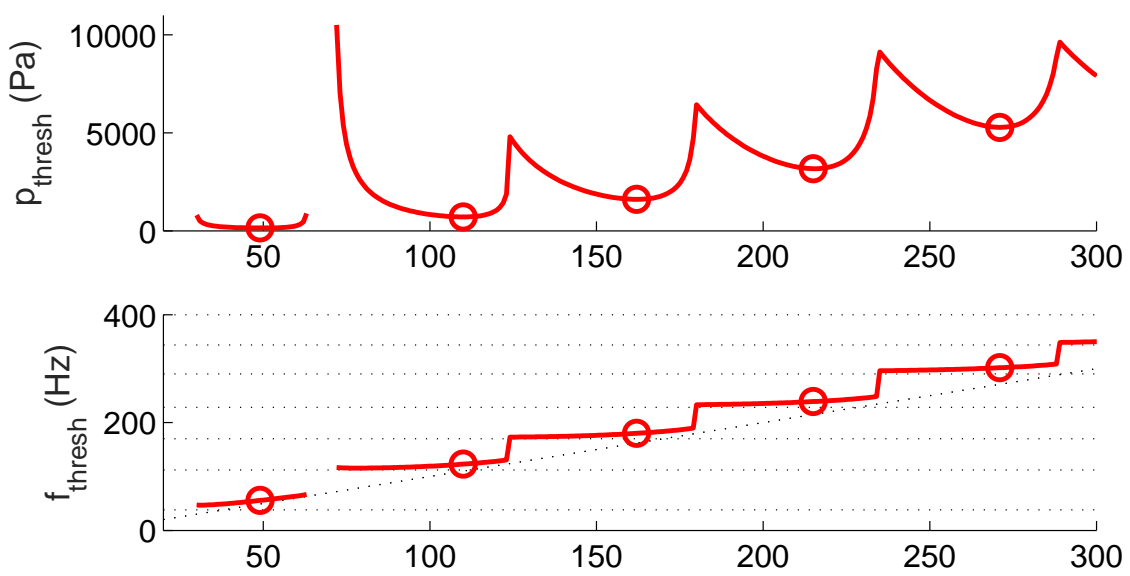

c)

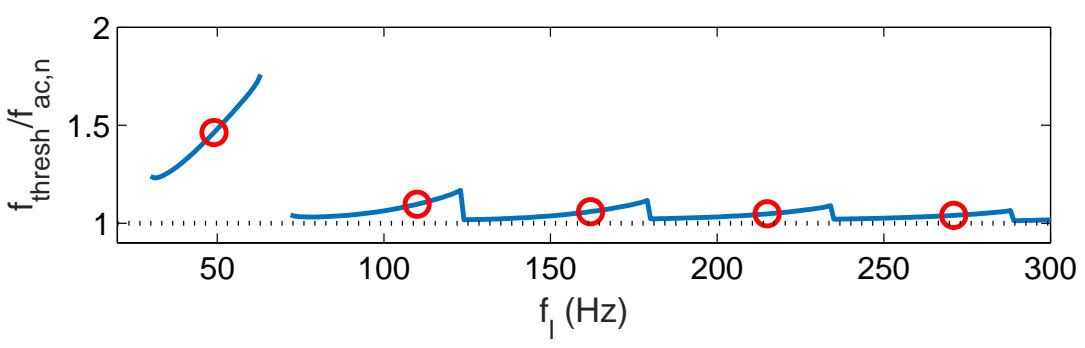

Figure 11: (colour online) Results of LSA (with lip parameters from Table 2) are plotted on (a) and (b) with a narrower $f_{l}$ range than in Figure 4. Horizontal dotted lines in (b) are the $f_{a c, n}$ values. The $f_{\text {thresh }}=f_{l}$ curve is also plotted (dotted). (c) is the $f_{\text {thresh }, n}^{\text {opt }} / f a c, n$ ratio. Circles indicate the $f_{l, n}^{o p t}$ lip resonance frequencies.

The LSA and the numerical simulation reveal a particularity of the trombone first regime: the oscillation frequency predicted by both methods is far above the acoustic resonance frequency, which results in a high $f_{\text {thresh }, n}^{\text {opt }} / f_{a c, n}$ ratio for $n=1$, while it is much smaller when $\mathrm{n} \geq 2$. This matches the experience of trombone players, who are able to play the pedal note in tune with the other notes, despite the large inharmonicity of the corresponding acoustic resonance. Therefore, a linearised model is able to predict a regime previously attributed to unexplained non-linear contributions of the upper acoustic resonances [Benade, 1976, p.405]. To complete this conclusion, LSA have been applied to the model with a resonator limited to the trombone's first mode. This results in $f_{\text {thresh }, 1}^{o p t}=61.06 \mathrm{~Hz}$. This confirms that the high $f_{\text {thresh }} / f_{a c, n}$ ratios are related to the first mode itself, and not to the contribution of the upper modes.

Bouasse proposed an experiment in which a trombone is played with a saxophone mouthpiece [Bouasse, 1986, p.370]. Gilbert and Aumond recently ran this experiment and published it [Gilbert and Aumond, 2008], together with audio and video recordings. The result is an instrument playing a low $E b 1$, that is, an oscillating frequency just under $f_{a c, 1}=38 \mathrm{~Hz}$, which is compatible with a playing frequency below the acoustic resonance frequency, characteristic of the inward-striking valve model used [Wilson and Beavers, 1974].

In order to explore the influence of nature of the exciter - inward-striking or outward striking this experiment is simulated here. A trombone is equipped with a saxophone mouthpiece instead 
of a trombone mouthpiece. The input impedance of this assembly is measured and fitted by a sum of complex modes, which is used as the resonator in the instrument model. The saxophone reed is modelled with an inward-striking valve as defined in [Wilson and Beavers, 1974] for example. Its equation is the same as Eq. (1) except the sign of the right-hand term: $\left(p_{b}-p(t)\right)$ becomes $\left(p(t)-p_{b}\right)$ in this model. The characteristics of the cane reed are taken from [Silva, 2009] : $f_{l}=1$ $\mathrm{kHz}, Q=1.1 ; 1 / \mu=4.9 \mathrm{~m}^{2} \cdot \mathrm{kg}^{-1} ; W=10^{-3} \mathrm{~m} ; h_{0}=5.10^{-4} \mathrm{~m}$. The results are presented in Figure 12.

The oscillating frequency of the simulated mouthpiece pressure is close to the first resonance frequency $f_{o s c} / f_{a c, 1}=0.99$ - a ratio contrasting with the high ratio obtained with an outwardstriking valve. The signal is nearly sinusoidal because of the lack of acoustic resonances matching the harmonics of this frequency in the impedance spectrum.

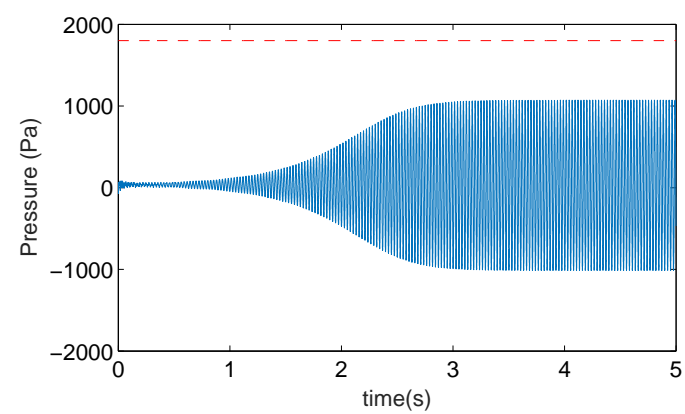

a)

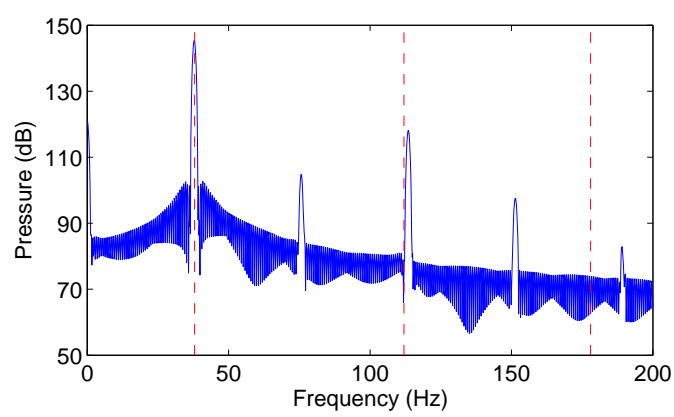

b)

Figure 12: (colour online) Results of simulation of a trombone with a tenor saxophone mouthpiece, modelled as an inward-striking valve with reed resonance frequency $f_{l}=1 \mathrm{kHz}, W=1 \mathrm{~cm}$, $h_{0}=5.10^{-4} \mathrm{~m}, Q_{l}=1.1,1 / \mu=4.9 \mathrm{~m}^{2} \cdot \mathrm{kg}^{-1}$. The blowing pressure $p_{b}=1800 \mathrm{~Pa}$ is slightly above $p_{\text {thresh }}=1760 \mathrm{~Pa}$. (a): blowing pressure (dashed line) and mouthpiece pressure (solid line). (b): spectrum of the mouthpiece pressure, showing an oscillation frequency $f_{\text {osc }}=37.85 \mathrm{~Hz}$ slightly below the first acoustic resonance $f_{a c, 1}=38 \mathrm{~Hz}$. Dashed lines represent the resonance frequencies of the bore.

Contrary to previous simulation, the $f_{o s c} / f_{a c, 1}$ ratio is very close to 1 . No pedal note phenomenon seems to be reproduced here. This simulation supports our choice of an outward-striking valve model, rather than an inward-striking one, to reproduce the behaviour of the lips for the trombone.

\subsection{A Saxhorn "ghost note" ?}

A complementary exploration is conducted on a Baritone-saxhorn in $B$ b. This instrument has a conical bore on almost its entire length, and it is played on the same range as the tenor trombone. Its acoustic resonance frequencies are quite similar to those of a trombone, as shown in Figure 13. The main difference between both instruments is the first resonance peak, which is nearly harmonic with the other ones on the saxhorn and very inharmonic on the trombone. Thus, unlike with the trombone, the pedal note $B b 1$ is close to the lowest resonance frequency. 

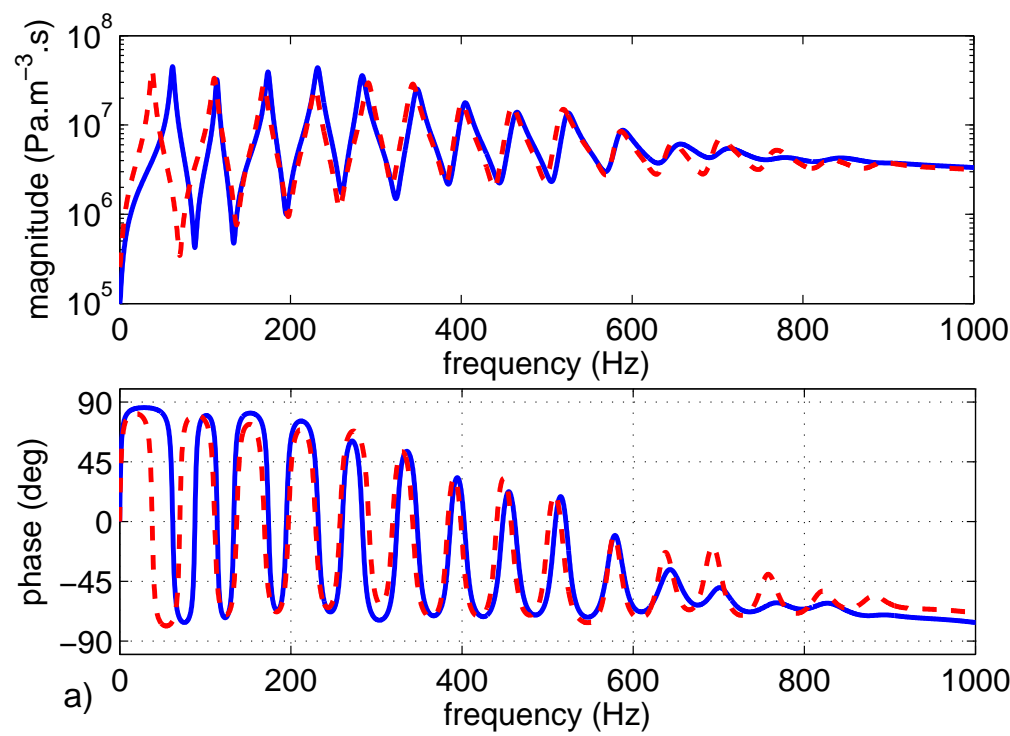

Figure 13: (colour online) Comparison between modal fits of the impedances of a trombone (dashed line) and of a saxhorn (solid line). The main difference in terms of frequency concerns the first peak.

The pedal note is easily playable by an intermediate musician. However, while practicing, the authors fortuitously found out another playable note, whose frequency lies between $f_{a c, 1}$ and $f_{a c, 2}$. Trials have been carried out on different saxhorn models and brands. The note played lies between $D b_{2}$ and $E b_{2}$, which means the frequency ratio $f_{o s c} / f_{a c, 1}$ lies between 1.19 and 1.35 . We call it the "ghost note" in this paper. Experienced saxhorn players further confirmed the existence, and facility of emission, of this ghost note on many different saxhorns and tubas.

LSA results on the saxhorn model are provided in Figure 14. The model used is similar to the trombone model, with $\mathrm{Z}$ equal to the input impedance of the saxhorn in Eq. (3). The behaviour is similar to that of the trombone, with a particularly high $f_{\text {thresh }, 1}^{o p t} / f_{a c, 1}$ ratio. Once again focusing on the $f_{\text {thresh }, n}^{o p t}$ values (circles in Figure 14), the ratio is $f_{\text {thresh }, 1}^{o p t} / f_{a c, 1}=1.23$. As in the case of the trombone, this ratio is smaller and quite constant for other modes $\left(f_{\text {thresh }, n}^{o p t} / f_{a c, n}<1.05, n \geq 2\right)$. Time-domain simulation of the saxhorn model on the first acoustic resonance (with $p_{b}=p_{\text {opt }, 1}+1 \%$, $f_{l}=f_{l, 1}^{o p t}$ and other parameters given in Table 2) confirms that $f_{o s c} / f_{a c, 1}=1.23$. 
a)
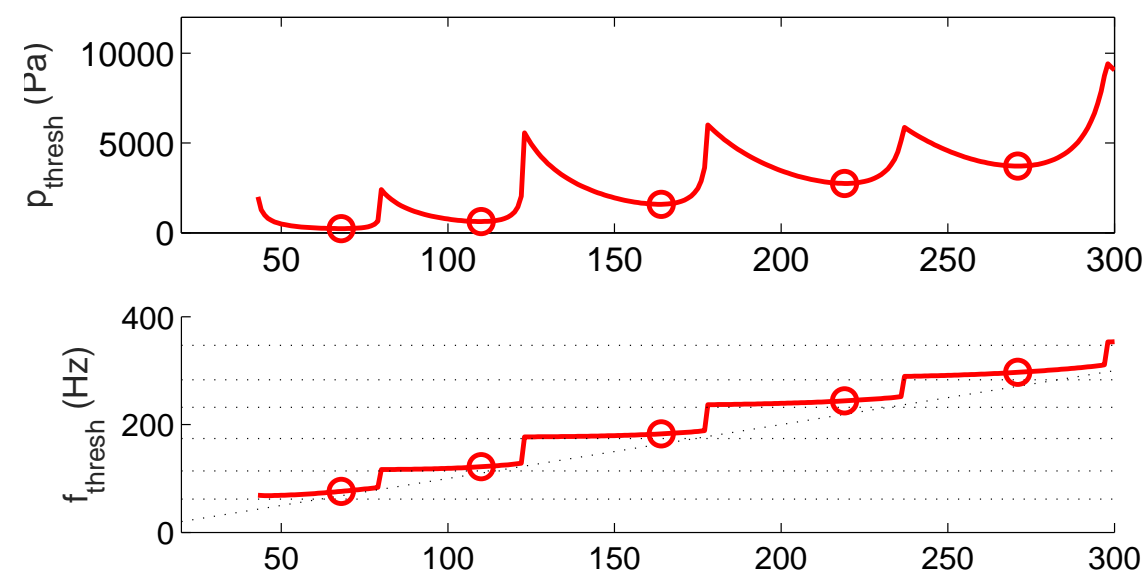

c)

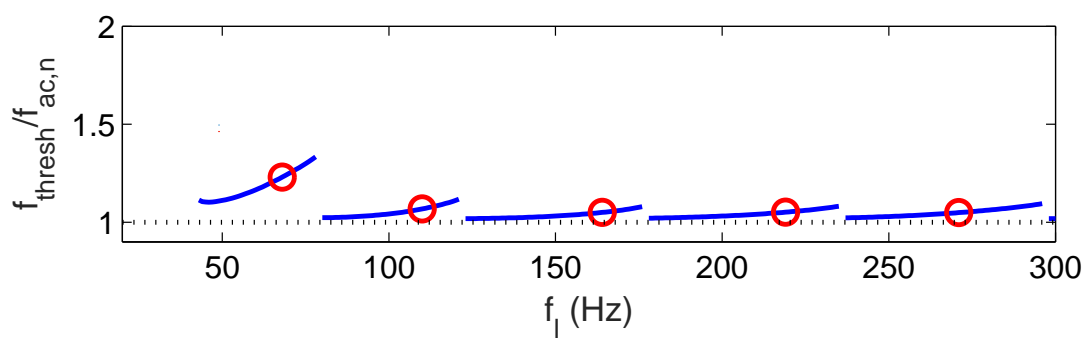

Figure 14: (colour online) LSA results for the saxhorn (with lip parameters given in Table 2) are given under the same form as those for the trombone in Figure 11. Circles indicate $p_{\text {opt }, n}$ (a) and $f_{\text {thresh,n }}^{\text {opt }}(\mathrm{b})$.

The gap between the lowest note played and the first acoustic resonance is smaller for the ghost note of the saxhorn $\left(f_{\text {thresh }, 1}^{\text {opt }} / f_{a c, 1}=1.23\right)$ than for the pedal note of the trombone. However, both are significantly higher than for the other modes $\left(f_{\text {thresh }, n}^{\text {opt }} / f_{a c, n} \leq 1.1\right.$ for $\left.n \geq 2\right)$. Another study [Velut et al., 2014] also highlights a high $f_{o s c} / f_{a c, 1}$ ratio for trombone and saxhorn despite quite different simulation conditions, which indicates the robustness of this phenomenon against changes in parameters. Thus, this simple linearised model makes it possible to predict the pedal note of the trombone and the ghost note of the saxhorn. However, a set of parameters simulating the pedal note $B b 1$ of the saxhorn with this model still needs to be found, should it exist.

\subsection{Shifting of the lowest resonance peak of the input impedances}

The trombone and the saxhorn are two examples of instruments having a high $f_{\text {thresh }, 1}^{o p t} / f_{a c, 1}$ ratio. The trombone has a higher ratio than the saxhorn, and the first bore resonance frequency is lower. To assess this negative correlation between $f_{a c, 1}$ and the $f_{t h r e s h, 1}^{o p t} / f_{a c, 1}$ ratio, the first resonance frequency of the input impedance is shifted for both instruments. This is done by modifying the $\left\{C_{1}, s_{1}\right\}$ values in Eq. (3) while keeping the other resonances unchanged, as well as the amplitude and quality factor of the first resonance.

For each value of $f_{a c, 1}$ tested, the $f_{\text {thresh }, 1}^{o p t} / f_{a c, 1}$ value is calculated. Results for both saxhorn and trombone are reported in Figure 15. For both instruments, the ratio increases when the first 
resonance frequency tends towards zero. Thus, as far as the studied model is concerned, the lower the resonance frequency, the larger the gap between the playing frequency and the first resonance frequency.

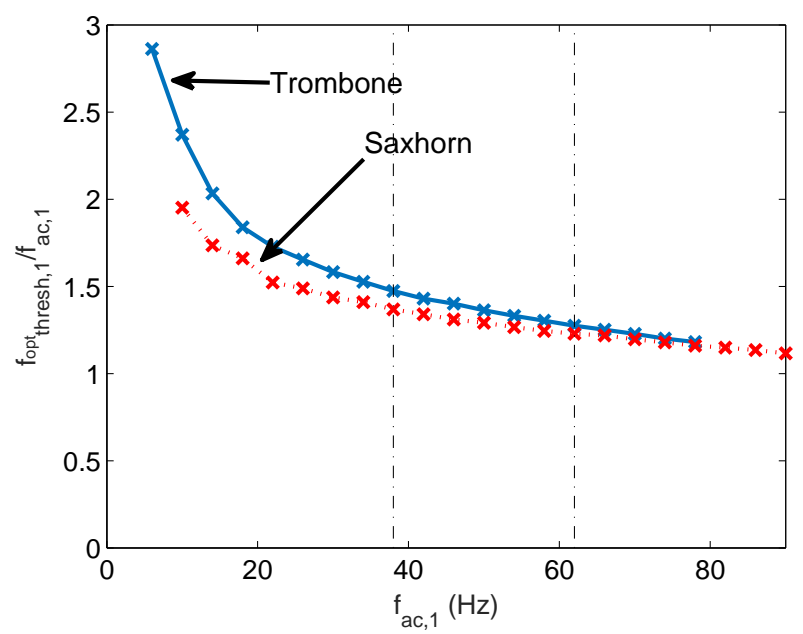

Figure 15: (colour online) Ratio between the predicted oscillation frequency $f_{\text {thresh }, 1}^{\text {opt }}$ and the acoustic resonance frequency $f_{a c, 1}$ for different values of the latter. The solid curve plots the results for the trombone, the dotted one for the saxhorn. Vertical dash-dotted lines are the original first resonance frequencies of a trombone $(38 \mathrm{~Hz})$ and a saxhorn $(62 \mathrm{~Hz})$.

\section{Conclusions}

Most results obtained in this study highlight the usefulness of Linear Stability Analysis (LSA) to understand various near-threshold behaviours of a complete nonlinear model of brass instrument applied to a trombone and a saxhorn.

The transient regimes, where the amplitude increases or decreases while staying close to the equilibrium solution, are perfectly predicted by LSA. Moreover, in time simulations, frequencies of periodic regimes measured in steady states are similar to those given by LSA, and close to the bore resonances. This remains true as long as the periodic regime emanating from the equilibrium solution remains stable. Indeed, once this periodic regime loses its stability, overblowing, quasi-periodicity or period-doubling occurs. Multiple instabilities of the equilibrium solution are shown by LSA, corresponding to several possible oscillation regimes, but this method will not determine on which of these regimes the system is going to oscillate. Further studies of the model with numerical continuation methods [Doedel, 1981, Cochelin and Vergez, 2009], should detect the bifurcations between oscillation branches and estimate the stability domain of each periodic solution, thus determining on which regime the system would oscillate. LSA is not adapted to detect quasi-periodicity and period-doubling.

The most striking results in this paper concern the lowest acoustic resonance of brass instruments. Indeed, in the case of the trombone, LSA predicts the production of the pedal note. LSA clearly indicates that for low enough acoustic resonance frequencies in the bore input impedance, the 
frequency of the emerging oscillation is far beyond the resonance frequency of the instrument. This allows the trombone pedal note to be played in tune, even though the corresponding resonance frequency is misaligned with the nearly harmonic series of the upper impedance peaks. This result from LSA is quite unexpected: the pedal note of the trombone seems to result from a coupling between the lips and the nearest acoustic mode below the playing frequency, just like for the other oscillation regimes. In contrast with previous studies, LSA show that higher bore resonances are not necessarily implied in the establishment of the pedal note, as shown by the simulation with a one-resonance bore. When applied to a saxhorn model, LSA shows that a note between $D b 2$ and $E b 2$ can be played, and this result is in agreement with what advanced players report. This note had, to our knowledge, never been documented before and is named "Ghost note".

However some questions are still unsolved. First of all, the reason why the ratio between the playing frequency at threshold and the acoustic resonance frequency rises when the latter decreases requires further attention. Moreover, neither LSA nor numerical simulations could explain the production of the saxhorn pedal note. This may be due to a limitation of the 1-DOF valve model for the lips or more simply to unsuitable parameter values. Indeed, in spite of the bibliographical review carried out for this study, choosing parameter values for a brass model remains challenging. Even though the results obtained look reasonable, i.e. consistent with musicians' experience, in vivo measurements of lip parameters during musical performance would be very valuable.

\section{Acknowledgments}

We wish to thank the ten skilled saxhorn players who confirmed the existence of the ghost note on different instruments. We also wish to thank Fabrice Silva for the discussions about our results and for his help in using MoReeSC.

Thanks to the reviewers, whose conscientious work improved the clarity of this article and raised some interesting questions.

This work was done in the framework of Labex MEX (ANR-10-LABX-0092) and of the project A*MIDEX (ANR-11-IDEX-0001-02), funded by the French National Research Agency (ANR). 


\section{A Equilibrium point of the system}

Prior to applying the linear stability analysis (LSA) to our model, the equilibrium solution must be computed before linearising the equations around this solution. This solution consists of a constant lip channel height $h(t)=h_{e}$, a constant flow between the lips $u_{e}$ and a constant pressure in the instrument $p(t)=p_{e}$. Finding these values consists in solving the equation system (6) with these constant values. With Eq. (5), the system becomes:

$$
\left\{\begin{array}{l}
0=-\omega_{l}^{2} h_{e}-\frac{p_{e}}{\mu}+\omega_{l}^{2} h_{0}+\frac{p_{b}}{\mu} \\
u_{e}=\sqrt{\frac{2}{\rho}} W h_{e} \sqrt{p_{b}-p_{e}} \\
0=Z_{c} C_{n} u_{e}+s_{n} p_{n e} \text { for } n \in[1, N] .
\end{array}\right.
$$

Considering the relation between $p(t)$ and its components $p_{n}(t)$, and adding the variable $A=$ $\sqrt{p_{b}-p_{e}}$, it becomes:

$$
\left\{\begin{array}{l}
h_{e}=h_{0}+\frac{A^{2}}{\mu \omega_{l}^{2}} \\
u_{e}=\sqrt{\frac{2}{\rho}} W h_{e} A \\
p_{e}=Z(\omega=0) u_{e} .
\end{array}\right.
$$

These three equations can now be combined:

$$
\frac{W Z(\omega=0)}{\mu \omega_{l}^{2}} \sqrt{\frac{2}{\rho}} A^{3}+A^{2}+W h_{0} Z(\omega=0) \sqrt{\frac{2}{\rho}} A-p_{b}=0,
$$

which leads to Eq. (9) given in Section 2.3.

\section{B Linearisation of Open-Loop Transfer Function}

This appendix details the calculations leading to the linearised expression of the open-loop transfer function of the model. The linearisation of the flow between lips $u$ simplifies to a ${ }^{\text {st }}$ degree Taylor expansion of Equation (5) near the equilibrium point:

$$
\tilde{u}(p, h)=u\left(p_{e}, h_{e}\right)-\left[\frac{\partial u}{\partial p}\left(p_{e}, h_{e}\right)\right]\left(\delta p(t)-\delta p_{e}\right)+\left[\frac{\partial u}{\partial h}\left(p_{e}, h_{e}\right)\right]\left(h(t)-h_{e}\right) .
$$

$\delta p=p_{b}-p(t)$ is the differential pressure through the lips. $\delta p_{e}$ and $h_{e}$ are the respective values of $\delta p$ and $h$ at the equilibrium solution. Similarly to the previous appendix A, the $p_{e}$ value is obtained by computing the roots of a $3^{\text {rd }}$ order polynomial whose variable is $A=\sqrt{\delta p}$ :

$$
A^{3}+\frac{A^{2}}{\beta}+\mu \cdot \omega_{l}^{2} \cdot h_{0} \cdot A-\frac{p_{b}}{\beta}=0 \quad \text { with } \beta=\frac{Z(\omega=0) \cdot W}{\mu \cdot \omega_{l}^{2}} \cdot \sqrt{\frac{2}{\rho}} .
$$

$8 h_{e}$ is given by Equation (1) in static conditions (all time derivatives being null): 


$$
h_{e}=h_{0}+\frac{\delta p_{e}}{\left(\mu \cdot \omega_{l}^{2}\right)} .
$$

All calculations being done, the linearised expression of the flow between the lips is:

$$
\tilde{u}(p, h)=W h_{e} \sqrt{\frac{2 \delta p_{e}}{\rho}}\left(\frac{\delta p(t)}{2 \delta p_{e}}+\frac{h(t)}{h_{e}}-\frac{1}{2}\right) .
$$

In the frequency domain, the equation of the lip movement (Equation (1)) gives the relation between the oscillating components of the mouthpiece pressure $P(\omega)$ and $H(\omega)$, the Fourier transform of $h(t)$. Since we are only interested in oscillating solutions, the $\omega=0$ case is ignored: hence, each constant value is omitted. This leads to the following equation:

$$
H(\omega)=D(\omega) \frac{P(\omega)}{\mu \cdot \omega_{l}^{2}}
$$

with $D(\omega)$ being the dynamics of the lips:

$$
D(\omega)=\frac{-1}{1-\frac{\omega^{2}}{\omega_{l}^{2}}+j \frac{\omega Q_{l}}{\omega_{l}}}
$$

which leads to this final expression of the linearized valve admittance, defined as $Y_{a}=\frac{\tilde{U}(\omega)}{P(\omega)}$ :

$$
Y_{a}=W \cdot h_{e} \cdot \sqrt{\frac{2 \delta p_{e}}{\rho}}\left(\frac{D(\omega)}{\mu \cdot \omega_{l}^{2} \cdot h_{e}}-\frac{1}{2 \delta p_{e}}\right) .
$$

Where $\tilde{U}(\omega)$ is the Fourier transform of the oscillating component of $\tilde{u}(t)$. Thus, constant values of Eq. (23) are also omitted. With this expression, $H_{O L}(\omega)=Y_{a}(\omega) \cdot Z(\omega)$ can be computed for section 3.4 .

\section{Nomenclature}

The symbols and abbreviations used all along this paper are recalled here, along with their meaning and the unit used:

- $h(t)$ : Height of the lip channel (m);

- $W$ : Width of the lip channel $(\mathrm{m})$;

- $h_{0}$ : Height of the lip channel at rest $(\mathrm{m})$;

- $\rho$ : Density of air at $20^{\circ} \mathrm{C}\left(\mathrm{kg} \cdot \mathrm{m}^{-3}\right)$;

- $\mu$ : Equivalent surfacic mass of the lips $\left(\mathrm{kg} \cdot \mathrm{m}^{-2}\right)$;

- $Q_{l}$ : Quality factor of the lips (no unit); 
- $p(t)$ and $P(\omega)$ : Waveform and Fourier transform of the pressure at the input of the bore of the instrument $(\mathrm{Pa})$;

- $p_{b}$ : Blowing pressure $(\mathrm{Pa})$;

- $p_{\text {thresh }}$ : Threshold value of $p_{b}$, above which the equilibrium solution is unstable $(\mathrm{Pa})$;

- $u(t)$ and $U(\omega)$ : Waveform and Fourier transform of the air flow at the input of the instrument $\left(\mathrm{m}^{3} \cdot \mathrm{s}^{-1}\right)$

- $Z(\omega)$ : Input impedance of the resonator $\left(\mathrm{Pa} \cdot \mathrm{m}^{-3} \cdot \mathrm{s}\right)$;

- $\omega_{l}=2 . \pi \cdot f_{l}$ : resonance frequency of the lips $\left(\mathrm{rad} \cdot \mathrm{s}^{-1}\right)$;

- $f_{\text {osc }}$ : Playing frequency of the instrument $(\mathrm{Hz})$;

- $f_{a c, n}$ : Acoustic resonance frequency of the $n^{\text {th }}$ mode $(\mathrm{Hz})$;

- $f_{\text {thresh }}$ : Oscillation frequency at $p_{\text {thresh }}(\mathrm{Hz})$.

- $p_{\text {opt }, n}$ : Lowest value of $p_{\text {thresh }}$ for the $n^{\text {th }}$ acoustic resonance (Pa);

- $f_{\text {thresh }, n}^{\text {opt }}$ : Value of $f_{\text {thresh }}(\mathrm{Hz})$ at $p_{b}=p_{\text {opt }, n}(\mathrm{~Hz})$;

- $f_{l, n}^{\text {opt }}$ : Value of $f_{l}(\mathrm{~Hz})$ at $p_{b}=p_{\text {opt }, n}(\mathrm{~Hz})$;

\section{References}

Adachi, S. and Sato, M. (1995). Time-domain simulation of sound production in the brass instrument. J. Acoust. Soc. Am., 97(6):3850-3861.

Adachi, S. and Sato, M. (1996). Trumpet sound simulation using a two-dimensional lip vibration model. J. Acoust. Soc. Am., 99(2):1200-1209.

Auvray, R., Fabre, B., and Lagrée, P. (2012). Regime change and oscillation thresholds in recorderlike instruments. J. Acoust. Soc. Am., 131(2):1574-1585.

Benade, A. (1976). Fundamentals of musical acoustics. Oxford Universitary Press, 608 pages.

Bergé, P., Pomeau, Y., and Vidal, C. (1995). L'ordre dans le chaos: vers une approche déterministe de la turbulence (Order in chaos : towards a deterministic approach of turbulence). Hermann, 352 pages.

Bergeot, B., Almeida, A., Vergez, C., and Gazengel, B. (2013). Prediction of the dynamic oscillation threshold in a clarinet model with a linearly increasing blowing pressure: influence of noise. Nonlinear Dynamics, 74(3):591-605. 
Bouasse, H. (1986). Instruments à vent-tome 1 (wind instruments-Volume 1). Librairie Scientifique et Technique Albert Blanchard, Paris, 411 pages.

Bouhuys, A. (1968). Pressure-flow events during wind instrument playing. Annals of New York Academy of Sciences, 155(1):264-275.

Boutin, H., Fletcher, N., Smith, J., and Wolfe, J. (2015). Relationship between pressure, flow, lip motion, and uptstream and downstream impedances for the trombone. J. Acoust. Soc. Am., 137(3):1195-1209.

Campbell, M. (2004). Brass instruments as we know them today. Acta Acustica united with Acustica, 90:600-610.

Campbell, M. and Greated, C. (1994). The musician's guide to Acoustics. Oxford Universitary Press, Oxford, 624 pages.

Chaigne, A. and Kergomard, J. (2016). Acoustics of Musical Instruments. Springer, 844 Pages.

Chang, Y. M. (1994). Reed stability. Journal of Fluids and Structures, 8:771-783.

Chen, J.-M., Smith, J., and Wolfe, J. (2011). Saxophonists tune vocal tract resonances in advanced performance techniques. J. Acoust. Soc. Am., 129:415-426.

Chen, J.-M., Smith, J., and Wolfe, J. (2012). Do trumpet players tune resonances of the vocal tract? J. Acoust. Soc. Am., 131(1):722-727.

Clinch, P., Troup, G., and Harris, L. (1982). The importance of the vocal tract resonance in clarinet and saxophone performance. Acustica, 50:280-284.

Cochelin, B. and Vergez, C. (2009). A high-order purely frequency-based harmonic balance formulation for continuation of periodic solutions. Journal of Sound and Vibration, 324.

Cullen, J., Gilbert, J., and Campbell, M. (2000). Brass instruments: Linear stability analysis and experiments with an artificial mouth. Acta Acustica united with Acustica, 86:704-724.

Dalmont, J.-P., Gazengel, B., Gilbert, J., and Kergomard, J. (1995). Some aspects of tuning and clean intonation in reed instruments. Applied Acoustics, 46(1):19-60.

Dalmont, J.-P., Gilbert, J., and Kergomard, J. (2000). Reed instruments, from small to large amplitude periodic oscillations and the Helmholtz motion analogy. Acta Acustica united with Acustica, 86(4):671-684.

Doedel, E. J. (1981). Auto: A program for the automatic bifurcation analysis of autonomous systems. In Proceedings of $10^{\text {th }}$ Manitoba conference on numerical mathematics and computation, pages 265-284, Winnipeg. 
Eliott, S. and Bowsher, J. (1982). Regeneration in brass wind instruments. Journal of Sound and Vibration, 83:181-217.

Farner, S., Vergez, C., Kergomard, J., and Lizée, A. (2006). Contribution to harmonic balance calculations of self-sustained periodic oscillations with focus on single-reed instruments. J. Acoust. Soc. Am., 119(3):1794-1804.

Ferrand, D., Vergez, C., and Silva, F. (2010). Seuils d'oscillation de la clarinette: validité de la représentation excitateur-résonateur (oscillation thresholds of the clarinet: validity of the exciterresonator representation). In Proceedings of $10^{\text {ème }}$ Congrès Français d'Acoustique.

Fletcher, N. (1993). Autonomous vibration of simple pressure-controlled valves in gas flows. $J$. Acoust. Soc. Am., 93(4):2172-2180.

Fréour, V. (2013). Acoustic and Respiratory Pressure Control in Brass Instrument Performance. PhD thesis, Departement of Music Research, Schulich School of Music, McGill University, Montreal, Canada.

Fréour, V., Lopes, N., Hélie, T., Caussé, R., and Scavone, G. (2015). In-vitro and numerical investigations of the influence of a vocal-tract resonance on lip auto-oscillations in trombone performance. Acta Acustica united with Acustica, 101:256-269.

Fréour, V. and Scavone, G. (2013). Acoustical interaction between vibrating lips, downstream air column, and upstream airways in trombone performance. J. Acoust. Soc. Am., 134(5):3887-3898.

Fritz, C. (2005). La clarinette et le clarinettiste: influence du conduit vocal sur la production du son (The clarinet and the player: role of the vocal tract on the sound production). PhD thesis, Université Paris 6 and University of New South Wales, France.

Gazengel, B., Guimezanes, T., Dalmont, J.-P., Doc, J., Fagart, S., and Léveillé, Y. (2007). Experimental investigation of the influence of the mechanical characteristics of the lip on the vibrations of the single reed. In Proceedings of ISMA 200\%, Barcelona.

Gibiat, V. (1988). Phase space representations of acoustical musical signals. Journal of Sound and Vibration, 123(3):539-536.

Gibiat, V. and Castellengo, M. (2000). Period doubling occurences in wind instruments musical performance. Acta Acustica united with Acustica, 86:746-756.

Gilbert, J. and Aumond, P. (2008). Pedal notes of brass instruments, a mysterious regime of oscillation. In Proceedings of Acoustics' 08, Paris.

Gilbert, J., Kergomard, J., and Ngoya, E. (1989). Calculation of the steady-state oscillations of a clarinet using the harmonic balance technique. J. Acoust. Soc. Am., 86(1):35-41. 
Grand, N., Gilbert, J., and Laloë, F. (1997). Oscillation threshold of woodwind instruments. Acustica, 83:137.

Guillemain, P., Vergez, C., Ferrand, D., and Farcy, A. (2010). An instrumented saxophone mouthpiece and its use to understand how an experienced musician plays. Acta Acustica United with Acustica, 96:622-634.

Hélie, T., Vergez, C., Lévine, J., and Rodet, X. (1999). Inversion of a physical model of a trumpet. In Proceedings of Conference on Decision and Control, pages 2593-2598, Phoenix, USA.

Helmholtz, H. (1877). On the Sensations of Tone. Dover Publications Inc., New York, 608 pages. Hirschberg, A., Gilbert, J., Msallam, R., and Wijnands, P. (1996). Shock waves in trombones. J. Acoust. Soc. Am., 99:1754.

Hirschberg, A., Kergomard, J., and Weinreich, G. (1995). Mechanics of musical instruments. Springer-Verlag, Wien, Austria.

Kaburagi, T., Yamada, N., Fukui, T., and Minamiya, E. (2011). A methodological and preliminary study on the acoustic effect of a trumpet player's vocal tract. J. Acoust. Soc. Am., $130(1): 536-545$.

Karkar, S., Vergez, C., and Cochelin, B. (2012). Oscillation threshold of a clarinet model: a numerical continuation approach. J. Acoust. Soc. Am., 131(1):698-707.

Kergomard, J., Dalmont, J., Gilbert, J., and Guillemain, P. (2004). Period doubling in cylindrical reed instruments. In Proceedings of the Joint Congress CFA/DAGA, Strasbourg, France.

Lopez, I., Hirschberg, A., Hirtum, A. V., Ruty, N., and Pelorson, X. (2006). Physical modeling of buzzing artificial lips: The effect of acoustical feedback. 92:1047-1059.

Macaluso, C. and Dalmont, J.-P. (2011). Trumpet with near-perfect harmonicity: Design and acoustic results. J. Acoust. Soc. Am, 129(1):404-414.

McIntyre, M., Schumacher, R., and Woodhouse, J. (1983). On the oscillations of musical instruments. J. Acoust. Soc. Am., 74(5):1325-1345.

MoReeSC (2013). Source website of the moreesc software. http://moreesc.lma.cnrs-mrs.fr/. Accessed: 2016-05-23.

Myers, A., Pyle, R., Gilbert, J., Campbell, M., Chick, J., and Logie, S. (2012). Effects of nonlinear sound propagation on the characteristic timbres of brass instruments. J. Acoust. Soc. Am., 131(1):678-688.

Neal, M., Richards, O., Campbell, D., and Gilbert, J. (2001). Study of the reed mechanism of brass instruments using an artificial mouth. In Proceedings of ISMA 2001, pages 99-102, Perugia, Italy. 
Newton, M. (2009). Experimental Mechanical and Fluid Mechanical Investigations of the Brass Instrument Lip-reed and the Human Vocal Folds. PhD thesis, University of Edinburgh.

Newton, M., Campbell, M., and Gilbert, J. (2008). Mechanical response measurements of real and artificial brass players lips. J. Acoust. Soc. Am., 123(1):EL14-EL20.

Ricaud, B., Guillemain, P., Kergomard, J., Silva, F., and Vergez, C. (2009). Behavior of reed woodwind instruments around the oscillation threshold. Acta Acustica united with Acustica, 95:733-743.

Richards, O. (2003). Investigation of the Lip Reed Using Computational Modelling and Experimental Studies with an Artificial Mouth. PhD thesis, University of Edimburgh, Edimburgh,Scottland.

Rodet, X. and Vergez, C. (1996). Physical models of trumpet-like instruments. detailed behavior and model improvements. In Proceedings of ICMC, pages 448-453, Hong-Kong.

Saneyoshi, J., Tenamura, H., and Yoshikawa, S. (1987). Feedback oscillations in reed woodwind and brasswind instruments. Acustica, 67:194-210.

Scavone, G., Lefebvre, A., and da Silva, A. (2008). Vocal tract interactions in saxophone performance. J. Acoust. Soc. Am., 123:3391.

Schumacher, R. (1981). Ab initio calculations of the oscillations of a clarinet. Acta Acustica united with Acustica, 48(2):71-85.

Silva, F. (2009). Émergence des auto-oscillations dans un instrument de musique à anche simple (Emergence of self-sustained oscillations in a single reed music instrument). PhD thesis, Universite de Provence Aix-Marseille 1.

Silva, F., Kergomard, J., and Vergez, C. (2007). Oscillation thresholds for "striking outwards" reeds coupled to a resonator. In Proceedings of ISMA 2007, Barcelona.

Silva, F., Kergomard, J., Vergez, C., and Gilbert, J. (2008). Interaction of reed and acoustic resonator in clarinet-like systems. J. Acoust. Soc. Am., 124(5):3284-3295.

Silva, F., Vergez, C., Guillemain, P., Kergomard, J., and Debut, V. (2014). MoReeSC : a framework for the simulation and analysis of sound production in reed and brass instruments. Acta Acustica United with Acustica, 100(1):126-138.

Taillard, P., Kergomard, J., and Laloë, F. (2010). Iterated maps for clarinet-like systems. Nonlinear Dynamics, 62:253-271.

Terrien, S., Vergez, C., and Fabre, B. (2014). To what extent can a linear analysis predict the behaviour of a flute model? In Proceedings of International Symposium on Musical Acoustics, Le Mans, France. 
Velut, L., Vergez, C., Gilbert, J., and Silva, F. (2014). Numerical simulation of the production of pedal notes in brass instruments. In Proceedings of International Symposium on Musical Acoustics, Le Mans, France.

Vergez, C. and Rodet, X. (1997). Model of the trumpet functionning: Real-time simulation and experiments with an artificial mouth model. In Proc. of International Symposium on Musical Acoustics (ISMA) 1997, Edimburgh, Scottland.

Wall, R. W. (2003). Simple methods for detecting zero crossing. In Proceedings of $29^{\text {th }}$ annual conference of the IEEE Industrial Electronics Society, pages 1-5, Roanoke, Virginia, USA. Paper no. 291.

Wangenheim, L. (2011). On the Barkhausen and Nyquist stability criteria. Analog. Integr. Circ. Sig. Process., 66:139-141.

Wilson, T. and Beavers, G. (1974). Operating modes of the clarinet. J. Acoust. Soc. Am., $56(2): 653-658$.

Yoshikawa, S. (1995). Acoustical behavior of brass player's lips. J. Acoust. Soc. Am, 97(3):19291939. 